\title{
Article \\ Discovery of Three Novel Cytospora Species in Thailand and Their Antagonistic Potential
}

\author{
Jutamart Monkai ${ }^{1,2}$, Saowaluck Tibpromma ${ }^{3,4}$, Areerat Manowong ${ }^{1}$, Ausana Mapook ${ }^{1} \oplus$, \\ Chada Norphanphoun ${ }^{1}$, Kevin D. Hyde ${ }^{1,5}$ and Itthayakorn Promputtha ${ }^{2,6, *(D)}$ \\ 1 Center of Excellence in Fungal Research, Mae Fah Luang University, Chiang Rai 57100, Thailand; \\ mjutamart@gmail.com (J.M.); areerat.man@mfu.ac.th (A.M.); phung.ausana@gmail.com (A.M.); \\ oomchn@gmail.com (C.N.); kdhyde3@gmail.com (K.D.H.) \\ 2 Department of Biology, Faculty of Science, Chiang Mai University, Chiang Mai 50200, Thailand \\ 3 Center for Mountain Futures, Kunming Institute of Botany, Kunming 650201, China; saowaluckfai@gmail.com \\ 4 CIFOR-ICRAF China Program, World Agroforestry (ICRAF), Kunming 650201, China \\ 5 Innovative Institute of Plant Health, Zhongkai University of Agriculture and Engineering, Haizhu District, \\ Guangzhou 510225, China \\ 6 Environmental Science Research Center, Faculty of Science, Chiang Mai University, \\ Chiang Mai 50200, Thailand \\ * Correspondence: itthayakorn.p@cmu.ac.th
}

Citation: Monkai, J.; Tibpromma, S.; Manowong, A.; Mapook, A.;

Norphanphoun, C.; Hyde, K.D.; Promputtha, I. Discovery of Three Novel Cytospora Species in Thailand and Their Antagonistic Potential. Diversity 2021, 13, 488. https:/ / doi.org/10.3390/d13100488

Academic Editor: Ipek Kurtboke

Received: 29 August 2021

Accepted: 28 September 2021

Published: 6 October 2021

Publisher's Note: MDPI stays neutral with regard to jurisdictional claims in published maps and institutional affiliations.

Copyright: (C) 2021 by the authors Licensee MDPI, Basel, Switzerland. This article is an open access article distributed under the terms and conditions of the Creative Commons Attribution (CC BY) license (https:// creativecommons.org/licenses/by/ $4.0 /)$.

\begin{abstract}
During an ongoing research survey of saprobic fungi in Thailand, four coelomycetous strains were isolated from decaying leaves in Chiang Mai and Phitsanulok Provinces. Morphological characteristics demonstrated that these taxa are typical of Cytospora in forming multi-loculate, entostromatic conidiomata, branched or unbranched conidiophores, with enteroblastic, phialidic conidiogenous cells and hyaline, allantoid, aseptate conidia. Multiloci phylogeny of ITS, LSU, ACT, RPB2, TEF1- $\alpha$ and TUB2 confirmed these taxa are distinct new species in Cytospora in Cytosporaceae (Diaporthales, Sordariomycetes), viz., Cytospora chiangmaiensis, C. phitsanulokensis and C. shoreae. Cytospora chiangmaiensis has a close phylogenetic relationship with $C$. shoreae, while C. phitsanulokensis is sister to $C$. acaciae. These three novel species were also preliminary screened for their antagonistic activity against five plant pathogenic fungi: Colletotrichum fructicola, Co. siamense, Co. artocarpicola, Co. viniferum and Fusarium sambucinum. Cytospora shoreae and C. phitsanulokensis showed $>60 \%$ inhibition against $C o$. viniferum and $F$. sambucinum, while $C$. chiangmaiensis had moderate inhibition activity against all pathogens.
\end{abstract}

Keywords: antifungal activity; coelomycetous fungi; Cytosporaceae; multiloci phylogeny; saprobes; taxonomy

\section{Introduction}

Cytospora Ehrenb. is mainly characterized by the coelomycetous asexual morph producing single or labyrinthine, loculate stromata, filamentous conidiophores, with enteroblastic, phialidic conidiogenous cells and hyaline, allantoid, aseptate conidia [1-3]. The sexual morph of Cytospora is characterized as solitary, immersed to erumpent ascostromata, composed of prosenchymatous or pseudoparenchymatous cells of valsoid or diatrypelloid configurations, embedded in ectostromatic disc, present or absent paraphyses, J-, ellipsoid to clavate asci with refractive apical ring, and hyaline, ellipsoid to allantoid, aseptate ascospores [1,4,5]. Ehrenberg [6] initially introduced the genus Cytospora to accommodate four species, C. betulina, C. epimyces, C. resinae and C. ribis. The type species, C. chrysosperma, was introduced by Donk [7] as the asexual morph of Valsa sordida [8]. Cytospora was previously treated as the asexual morph of Valsa and other related genera, Leucocytospora, Leucostoma, Valsella and Valseutypella $[4,8,9]$. Based on the International Code of Nomenclature for Algae, Fungi, and Plants (ICN) [10], Rossman et al. [8] recommended to use Cytospora instead of Valsa as Cytospora was prior introduced and thus Valsa was treated as 
a synonym of Cytospora, together with the genera Leucocytospora, Leucostoma, Valsella and Valseutypella $[4,8]$.

Species identification in Cytospora was problematic in the past due to overlapping morphological characteristics and wide host ranges [11-15]. A polyphasic approach including host association, geographical distribution, morphological characteristics and multiloci phylogeny has been widely accepted for identifying Cytospora species [1-3,5,9,16-18]. To date, 671 species epithets are listed in Index Fungorum [19], but less than a quarter of taxa in this genus are clarified with phylogenetic placement. In recent years, about 117 species have been accepted based on morpho-molecular approaches [20], and these species have primarily been described from China, Italy, Russia and Thailand [1-3,5,9,16-18,21].

Cytospora is a cosmopolitan genus with diverse ecological niches, including plant pathogens, endophytes and saprobes, colonizing decaying wood substrates $[1,2,17,18]$. Cytospora species are important causal agents of diebacks and stem cankers in numerous woody plants such as Betulaceae, Pinophyta, Fabaceae, Juglandaceae, Rosaceae, Salicaceae, Tiliaceae and Ulmaceae [1,3,16-18,22,23]. Furthermore, Cytospora has also been reported to produce potential secondary metabolites with various biological activities, such as antibacterial, antifungal and antiviral activities [24-26]. Investigation of the endophytic fungus Cytospora sp. from Costa Rica yielded novel antibiotic compounds: cytoskyrins and cytosporones $[24,25]$. Upon screening novel secondary metabolites produced by the endophytic fungus Cytospora sp. from Chinese mangrove, new bicyclic sesquiterpene and seiricardine $\mathrm{D}$ were isolated and they showed effective inhibition of human and plant pathogens [27].

During a survey of saprobic fungi in Thailand, three Cytospora species were collected and isolated. Based on morphological characteristics and phylogenetic analyses, our strains were identified as new species of Cytospora. In addition, these fungi were tested in vitro as the first step for screening new biocontrol agents against pathogenic fungi.

\section{Materials and Methods}

\subsection{Collection and Isolation of Fungi}

Decaying leaves were collected from disturbed forests in Chiang Mai and Phitsanulok Provinces in Thailand. The forests were disturbed due to the utilization of bioresources by the local communities. Samples were kept in plastic bags with labels of location, date, host and collector details before being taken to laboratory for morphological observation. We followed Senanayake et al. [28] for single spore isolation by using potato dextrose agar (PDA) and incubating at room temperature $\left(28^{\circ} \mathrm{C}\right)$. Pure cultures were deposited in Mae Fah Luang University Culture Collection (MFLUCC) and type specimens were deposited in the Herbarium of Mae Fah Luang University (MFLU). New taxa were registered in Faces of Fungi [29] and Index Fungorum databases [19].

\subsection{Morphological Observation}

Conidiomata on host surface were examined using a Motic SMZ 168 Series stereo microscope (Motic Incorporation Ltd., Hong Kong). Hand-sectioning of conidiomata was carried out and the sections were mounted on a slide with a drop of distilled water. Morphological characteristics including structure and size of stromata, ectostromatic disc, ostioles as well as shape and size of conidiogenous cells, conidiophores and conidia were observed and photographed using a Nikon ECLIPSE 80i compound microscope equipped with a Canon EOS 600D digital camera. Microscopic elements were measured using the Tarosoft (R) Image Frame Work program. The measurements of each structure were represented as minimum value-maximum value $(\bar{x}=$ sum of all measurements $/ n$, $n=$ number of measurements). The figures were processed using Adobe Photoshop CS6 Extended version 10.0 software (Adobe Systems, San Jose, CA, USA). 


\subsection{DNA Extraction, PCR Amplification and Sequencing}

Genomic DNA was extracted from fresh mycelium which was grown on PDA for 1-2 weeks using the Biospin Fungus Genomic DNA Extraction Kit (BioFlux ${ }^{\circledR}$, Hangzhou, China) following the manufacturer's protocol. The amplification of specific ribosomal DNA regions was carried out using two gene regions, including the internal transcribed spacers region of ribosomal DNA (ITS) [30] and the partial 28S large subunit nuclear ribosomal DNA (LSU) [31], and four protein coding gene regions: the RNA polymerase II second largest subunit (RPB2) [32], $\alpha$-actin (ACT) [33], the translation elongation factor $1-\alpha$ (TEF1- $\alpha$ ) [33] and beta-tubulin (TUB2) [34]. The final volume of PCR mixtures was $25 \mu \mathrm{L}$,

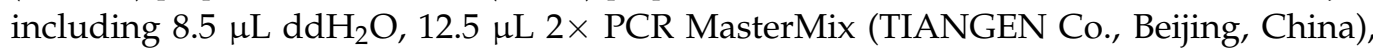
$2 \mu \mathrm{L}$ DNA template and $1 \mu \mathrm{L}$ of each forward and reverse primer. The PCR primers and conditions for each gene regions are described in Table 1 . The purification and sequencing of PCR products were conducted by TsingKe Company (Kunming, China).

Table 1. Partial marker regions, primers and PCR conditions used in this study.

\begin{tabular}{|c|c|c|}
\hline Genes (Primer Pair) & References & PCR Condition \\
\hline $\begin{array}{l}\text { ITS (ITS5/ITS4) } \\
\text { LSU (LROR/LR5) } \\
\text { TEF1- } \alpha(728 F / 986 R) \\
\text { TUB2 (Bt2a/Bt2b) }\end{array}$ & $\begin{array}{l}{[30]} \\
{[31]} \\
{[33]} \\
{[34]}\end{array}$ & $\begin{array}{l}\text { An initial denaturation step of } 3 \mathrm{~min} \text { at } 94{ }^{\circ} \mathrm{C} \text {, followed by } \\
35 \text { cycles of } 30 \mathrm{~s} \text { at } 94{ }^{\circ} \mathrm{C}, 50 \mathrm{~s} \text { at } 55^{\circ} \mathrm{C} \text { and } 90 \mathrm{~s} \text { at } 72^{\circ} \mathrm{C} \text {, and } \\
\text { a final extension step of } 10 \mathrm{~min} \text { at } 72{ }^{\circ} \mathrm{C}\end{array}$ \\
\hline $\mathrm{ACT}(512 \mathrm{~F} / 783 \mathrm{R})$ & [33] & $\begin{array}{l}\text { An initial denaturation step of } 5 \mathrm{~min} \text { at } 96^{\circ} \mathrm{C} \text {, followed by } \\
35 \text { cycles of } 40 \mathrm{~s} \text { at } 95^{\circ} \mathrm{C}, 30 \mathrm{~s} \text { at } 58^{\circ} \mathrm{C} \text { and } 1 \mathrm{~min} \text { at } 72^{\circ} \mathrm{C} \text {, } \\
\text { and a final extension step of } 5 \mathrm{~min} \text { at } 72^{\circ} \mathrm{C}\end{array}$ \\
\hline RPB2 (fRPB2-5F / fRPB2-7cR) & {$[32]$} & $\begin{array}{l}\text { An initial denaturation step of } 5 \mathrm{~min} \text { at } 95^{\circ} \mathrm{C} \text {, followed by } \\
40 \text { cycles of } 1 \mathrm{~min} \text { at } 95{ }^{\circ} \mathrm{C}, 1 \mathrm{~min} \text { at } 52{ }^{\circ} \mathrm{C} \text { and } 90 \mathrm{~s} \text { at } 72{ }^{\circ} \mathrm{C} \text {, } \\
\text { and a final extension step of } 10 \mathrm{~min} \text { at } 72^{\circ} \mathrm{C}\end{array}$ \\
\hline
\end{tabular}

\subsection{Phylogenetic Analyses}

Sequences generated in this study were analyzed with other sequences retrieved from the GenBank (www.ncbi.nlm.nih.gov/blast/, accessed on 1 August 2021) and recent publications $[1,2,17]$ (Table 2). Alignments of the individual locus were aligned with MAFFT v.7 (http:/ / mafft.cbrc.jp/alignment/server/index.html, accessed on 3 August 2021; [35]) and automatically trimmed using TrimAl (http:/ / trimal.cgenomics.org accessed on 1 August 2021; [36]). The single-locus datasets of ITS, LSU, ACT, RPB2, TEF1- $\alpha$ and TUB2 regions were initially analyzed by maximum likelihood (ML) criterion with 120,72 , $69,50,55$ and 41 sequences, respectively. The tree topologies obtained from single-locus analyses were checked for incongruence and the results did not show any topological conflicts. Phylogenetic analyses of the final concatenated ITS, LSU, ACT, RPB2, TEF1- $\alpha$ and TUB2 sequence datasets were performed via maximum likelihood (ML), maximum parsimony (MP) and Bayesian inference (BI).

Table 2. Taxa used in the phylogenetic analyses and their corresponding GenBank accession numbers. The newly generated sequences are indicated in red bold. The ex-type strains are bold. N/A = unavailable sequence data.

\begin{tabular}{|c|c|c|c|c|c|c|c|}
\hline \multirow{2}{*}{ Species Name } & \multirow{2}{*}{$\begin{array}{c}\text { Culture } \\
\text { Accession No. }\end{array}$} & \multicolumn{6}{|c|}{ Genbank Accession No. } \\
\hline & & ITS & LSU & ACT & RPB2 & TEF1- $\alpha$ & TUB2 \\
\hline Cytospora abyssinica & CMW 10181 & AY347353 & $\mathrm{N} / \mathrm{A}$ & $\mathrm{N} / \mathrm{A}$ & $\mathrm{N} / \mathrm{A}$ & $\mathrm{N} / \mathrm{A}$ & $\mathrm{N} / \mathrm{A}$ \\
\hline C. abyssinica & CMW 10178 & AY347354 & $\mathrm{N} / \mathrm{A}$ & $\mathrm{N} / \mathrm{A}$ & $\mathrm{N} / \mathrm{A}$ & $\mathrm{N} / \mathrm{A}$ & $\mathrm{N} / \mathrm{A}$ \\
\hline C. abyssinica & CMW 10179 & AY347352 & $\mathrm{N} / \mathrm{A}$ & $\mathrm{N} / \mathrm{A}$ & $\mathrm{N} / \mathrm{A}$ & $\mathrm{N} / \mathrm{A}$ & $\mathrm{N} / \mathrm{A}$ \\
\hline C. abyssinica & CBS 117004 & KY051833 & KX965299 & KX964737 & KX965495 & KX965098 & $\mathrm{N} / \mathrm{A}$ \\
\hline C. acaciae & CBS 468.69 & DQ243804 & $\mathrm{N} / \mathrm{A}$ & $\mathrm{N} / \mathrm{A}$ & $\mathrm{N} / \mathrm{A}$ & $\mathrm{N} / \mathrm{A}$ & $\mathrm{N} / \mathrm{A}$ \\
\hline
\end{tabular}


Table 2. Cont.

\begin{tabular}{|c|c|c|c|c|c|c|c|}
\hline \multirow{2}{*}{ Species Name } & \multirow{2}{*}{$\begin{array}{c}\text { Culture } \\
\text { Accession No. }\end{array}$} & \multicolumn{6}{|c|}{ Genbank Accession No. } \\
\hline & & ITS & LSU & $\mathrm{ACT}$ & RPB2 & TEF1- $\alpha$ & TUB2 \\
\hline C. acaciae & Fi2427 & MG253918 & $\mathrm{N} / \mathrm{A}$ & $\mathrm{N} / \mathrm{A}$ & $\mathrm{N} / \mathrm{A}$ & $\mathrm{N} / \mathrm{A}$ & $\mathrm{N} / \mathrm{A}$ \\
\hline C. acaciae & СРC 28392 & KY051980 & KX965438 & KX964858 & KX965579 & KX965220 & KX965027 \\
\hline C. acaciae & CBS 468.69 & KY051937 & KX965394 & KX964817 & $\mathrm{N} / \mathrm{A}$ & KX965181 & KX964990 \\
\hline C. acaciae & CBS 362.93 & KY051929 & KX965386 & KX964811 & KX965547 & KX965173 & KX964983 \\
\hline C. acaciae & CBS 112156 & KY051776 & KX965241 & KX964688 & $\mathrm{N} / \mathrm{A}$ & $\mathrm{N} / \mathrm{A}$ & KX964888 \\
\hline C. amygdali & CBS 144233 & MG971853 & $\mathrm{N} / \mathrm{A}$ & MG972002 & $\mathrm{N} / \mathrm{A}$ & MG971659 & $\mathrm{N} / \mathrm{A}$ \\
\hline C. amygdali & LH356 & MG971852 & $\mathrm{N} / \mathrm{A}$ & MG972001 & $\mathrm{N} / \mathrm{A}$ & MG971658 & $\mathrm{N} / \mathrm{A}$ \\
\hline C. brevispora & CBS 116829 & AF192321 & $\mathrm{N} / \mathrm{A}$ & N/A & $\mathrm{N} / \mathrm{A}$ & N/A & $\mathrm{N} / \mathrm{A}$ \\
\hline C. brevispora & CBS 116811 & AF192315 & $\mathrm{N} / \mathrm{A}$ & $\mathrm{N} / \mathrm{A}$ & $\mathrm{N} / \mathrm{A}$ & $\mathrm{N} / \mathrm{A}$ & $\mathrm{N} / \mathrm{A}$ \\
\hline C. brevispora & CBS 116829 & KY051803 & KX965267 & KX964709 & KX965477 & KX965073 & KX964909 \\
\hline C. brevispora & CBS 116813 & KY051788 & KX965252 & KX964698 & KX965470 & KX965061 & KX964898 \\
\hline C. californica & CBS 144234 & MG971935 & $\mathrm{N} / \mathrm{A}$ & MG972083 & $\mathrm{N} / \mathrm{A}$ & MG971645 & $\mathrm{N} / \mathrm{A}$ \\
\hline C. californica & KARE1106 & MG971948 & $\mathrm{N} / \mathrm{A}$ & MG972094 & $\mathrm{N} / \mathrm{A}$ & MG971647 & $\mathrm{N} / \mathrm{A}$ \\
\hline C. californica & KARE93 & MG971930 & $\mathrm{N} / \mathrm{A}$ & MG972079 & $\mathrm{N} / \mathrm{A}$ & MG971640 & $\mathrm{N} / \mathrm{A}$ \\
\hline C. cedri & CBS 196.50 & AF192311 & $\mathrm{N} / \mathrm{A}$ & $\mathrm{N} / \mathrm{A}$ & $\mathrm{N} / \mathrm{A}$ & $\mathrm{N} / \mathrm{A}$ & $\mathrm{N} / \mathrm{A}$ \\
\hline C. cedri & CBS 196.50 & KY051905 & KX965364 & KX964790 & KX965534 & KX965153 & $\mathrm{N} / \mathrm{A}$ \\
\hline C. ceratosperma & CBS 116.21 & AY347335 & $\mathrm{N} / \mathrm{A}$ & $\mathrm{N} / \mathrm{A}$ & N/A & N/A & N/A \\
\hline C. ceratosperma & CFCC 89625 & KR045646 & KR045725 & $\mathrm{N} / \mathrm{A}$ & KU710977 & KР310861 & KR045687 \\
\hline C. ceratospermopsis & CFCC 89626 & KR045647 & KR045726 & KU711011 & KU710978 & KU710934 & KR045688 \\
\hline C. ceratospermopsis & CFCC 89627 & KR045648 & KR045727 & KU711012 & KU710979 & KU710935 & KR045689 \\
\hline C. chiangmaiensis & $\begin{array}{l}\text { MFLUCC } \\
21-0049\end{array}$ & MZ356514 & MZ356518 & MZ451157 & MZ451165 & MZ451161 & MZ451169 \\
\hline C. curvata & $\begin{array}{l}\text { MFLUCC } \\
15-0865\end{array}$ & KY417728 & $\mathrm{N} / \mathrm{A}$ & KY417694 & $\mathrm{N} / \mathrm{A}$ & $\mathrm{N} / \mathrm{A}$ & $\mathrm{N} / \mathrm{A}$ \\
\hline C. davidiana & CXY 1350 & KM034870 & $\mathrm{N} / \mathrm{A}$ & $\mathrm{N} / \mathrm{A}$ & $\mathrm{N} / \mathrm{A}$ & $\mathrm{N} / \mathrm{A}$ & KM034902 \\
\hline C. davidiana & CXY 1374 & KM034869 & $\mathrm{N} / \mathrm{A}$ & $\mathrm{N} / \mathrm{A}$ & $\mathrm{N} / \mathrm{A}$ & $\mathrm{N} / \mathrm{A}$ & KM034901 \\
\hline C. diopuiensis & $\begin{array}{l}\text { MFLUCC } \\
18-1419\end{array}$ & MK912137 & MK571765 & MN685819 & $\mathrm{N} / \mathrm{A}$ & $\mathrm{N} / \mathrm{A}$ & $\mathrm{N} / \mathrm{A}$ \\
\hline C. diopuiensis & $\begin{array}{l}\text { MFLUCC } \\
18-0598\end{array}$ & MT215491 & MT215540 & $\mathrm{N} / \mathrm{A}$ & MT212203 & $\mathrm{N} / \mathrm{A}$ & $\mathrm{N} / \mathrm{A}$ \\
\hline C. erumpens & $\begin{array}{l}\text { MFLUCC } \\
16-0580\end{array}$ & KY417733 & KY417767 & KY417699 & KY417801 & $\mathrm{N} / \mathrm{A}$ & $\mathrm{N} / \mathrm{A}$ \\
\hline C. erumpens & CFCC 53163 & MK673059 & MK673089 & MK673029 & MK673000 & MK672948 & MK672975 \\
\hline C. eucalypti & LSEQ & AY347340 & $\mathrm{N} / \mathrm{A}$ & $\mathrm{N} / \mathrm{A}$ & $\mathrm{N} / \mathrm{A}$ & $\mathrm{N} / \mathrm{A}$ & $\mathrm{N} / \mathrm{A}$ \\
\hline C. eucalypti & CBS 144241 & MG971907 & $\mathrm{N} / \mathrm{A}$ & MG972056 & $\mathrm{N} / \mathrm{A}$ & MG971617 & $\mathrm{N} / \mathrm{A}$ \\
\hline C. eucalypti & $7 \mathrm{G}-62$ & MG971910 & $\mathrm{N} / \mathrm{A}$ & MG972060 & $\mathrm{N} / \mathrm{A}$ & MG971620 & N/A \\
\hline C. eucalypti & CBS 116814 & KY051789 & KX965253 & KX964699 & $\mathrm{N} / \mathrm{A}$ & KX965062 & KX964899 \\
\hline C. eucalyptina & CMW 5882 & AY347375 & $\mathrm{N} / \mathrm{A}$ & $\mathrm{N} / \mathrm{A}$ & $\mathrm{N} / \mathrm{A}$ & N/A & N/A \\
\hline C. eucalyptina & CBS 118085 & KY051848 & KX965315 & $\mathrm{N} / \mathrm{A}$ & $\mathrm{N} / \mathrm{A}$ & $\mathrm{N} / \mathrm{A}$ & KX964935 \\
\hline C. eucalyptina & CBS 116853 & KY051822 & KX965288 & KX964727 & $\mathrm{N} / \mathrm{A}$ & $\mathrm{N} / \mathrm{A}$ & KX964918 \\
\hline C. fraxinigena & MFLU 17-0880 & MF190134 & MF190079 & N/A & $\mathrm{N} / \mathrm{A}$ & $\mathrm{N} / \mathrm{A}$ & $\mathrm{N} / \mathrm{A}$ \\
\hline C. fraxinigena & $\begin{array}{l}\text { MFLUCC } \\
14-0868\end{array}$ & MF190133 & MF190078 & $\mathrm{N} / \mathrm{A}$ & $\mathrm{N} / \mathrm{A}$ & $\mathrm{N} / \mathrm{A}$ & $\mathrm{N} / \mathrm{A}$ \\
\hline C. gigaspora & CFCC 50014 & KR045630 & KR045710 & KU710999 & KU710959 & KU710922 & KR045671 \\
\hline C. gigaspora & CFCC 89634 & KF765671 & KF765687 & KU711000 & KU710960 & KU710923 & KR045672 \\
\hline C. italica & $\begin{array}{l}\text { MFLUCC } \\
14-0440\end{array}$ & KU900329 & KU900301 & $\mathrm{N} / \mathrm{A}$ & $\mathrm{N} / \mathrm{A}$ & $\mathrm{N} / \mathrm{A}$ & $\mathrm{N} / \mathrm{A}$ \\
\hline C. junipericola & BBH 42444 & MF190126 & MF190071 & $\mathrm{N} / \mathrm{A}$ & $\mathrm{N} / \mathrm{A}$ & MF377579 & $\mathrm{N} / \mathrm{A}$ \\
\hline C. junipericola & MFLU 17-0882 & MF190125 & MF190072 & $\mathrm{N} / \mathrm{A}$ & $\mathrm{N} / \mathrm{A}$ & MF377580 & $\mathrm{N} / \mathrm{A}$ \\
\hline C. kunzei & CBS 118556 & DQ243791 & $\mathrm{N} / \mathrm{A}$ & N/A & $\mathrm{N} / \mathrm{A}$ & $\mathrm{N} / \mathrm{A}$ & N/A \\
\hline C. kunzei & CBS 118093 & KY051855 & KX965322 & KX964754 & $\mathrm{N} / \mathrm{A}$ & KX965116 & KX964941 \\
\hline C. kunzei & CBS 114651 & KY051780 & KX965243 & KX964691 & $\mathrm{N} / \mathrm{A}$ & KX965055 & KX964890 \\
\hline C. leucostoma & CFCC 50023 & KR045635 & KR045715 & KU711003 & KU710964 & KU710926 & KR045676 \\
\hline C. leucostoma & CFCC 53156 & MN854447 & MN854658 & MN850762 & MN850748 & MN850755 & MN861117 \\
\hline C. leucostoma & $\begin{array}{l}\text { MFLUCC } \\
16-0574\end{array}$ & KY417731 & KY417764 & KY417696 & KY417798 & $\mathrm{N} / \mathrm{A}$ & $\mathrm{N} / \mathrm{A}$ \\
\hline
\end{tabular}


Table 2. Cont.

\begin{tabular}{|c|c|c|c|c|c|c|c|}
\hline \multirow{2}{*}{ Species Name } & \multirow{2}{*}{$\begin{array}{c}\text { Culture } \\
\text { Accession No. }\end{array}$} & \multicolumn{6}{|c|}{ Genbank Accession No. } \\
\hline & & ITS & LSU & $\mathrm{ACT}$ & RPB2 & TEF1- $\alpha$ & TUB2 \\
\hline C. lumnitzericola & $\begin{array}{l}\text { MFLUCC } \\
17-0508\end{array}$ & MG975778 & MH253453 & MH253457 & MH253461 & $\mathrm{N} / \mathrm{A}$ & $\mathrm{N} / \mathrm{A}$ \\
\hline C. magnoliae & IMI259790 & JX438623 & $\mathrm{N} / \mathrm{A}$ & $\mathrm{N} / \mathrm{A}$ & $\mathrm{N} / \mathrm{A}$ & JX438565 & $\mathrm{N} / \mathrm{A}$ \\
\hline C. mali-spectabilis & CFCC 53181 & MK673066 & MK673096 & MK673036 & MK673006 & MK672953 & MK672982 \\
\hline C. multicollis & CBS 105.89 & DQ243803 & $\mathrm{N} / \mathrm{A}$ & $\mathrm{N} / \mathrm{A}$ & $\mathrm{N} / \mathrm{A}$ & $\mathrm{N} / \mathrm{A}$ & $\mathrm{N} / \mathrm{A}$ \\
\hline C. nitschkii & CMW 10180 & AY347356 & $\mathrm{N} / \mathrm{A}$ & $\mathrm{N} / \mathrm{A}$ & $\mathrm{N} / \mathrm{A}$ & $\mathrm{N} / \mathrm{A}$ & $\mathrm{N} / \mathrm{A}$ \\
\hline C. nitschkii & CMW 10184 & AY347355 & $\mathrm{N} / \mathrm{A}$ & $\mathrm{N} / \mathrm{A}$ & $\mathrm{N} / \mathrm{A}$ & $\mathrm{N} / \mathrm{A}$ & $\mathrm{N} / \mathrm{A}$ \\
\hline C. nitschkii & CBS 130.95 & KY051879 & KX965344 & $\mathrm{N} / \mathrm{A}$ & $\mathrm{N} / \mathrm{A}$ & $\mathrm{N} / \mathrm{A}$ & $\mathrm{N} / \mathrm{A}$ \\
\hline C. nitschkii & CBS 116854 & MH863003 & MH874559 & KX964728 & KX965487 & KX965090 & $\mathrm{N} / \mathrm{A}$ \\
\hline C. nivea & $\begin{array}{l}\text { MFLUCC } \\
15-0860\end{array}$ & KY417737 & KY417771 & KY417703 & KY417805 & $\mathrm{N} / \mathrm{A}$ & $\mathrm{N} / \mathrm{A}$ \\
\hline C. nivea & CFCC 89641 & KF765683 & KF765699 & KU711006 & KU710967 & KU710929 & KR045679 \\
\hline C. notastroma & $\mathrm{K} 3$ & JX438631 & $\mathrm{N} / \mathrm{A}$ & $\mathrm{N} / \mathrm{A}$ & $\mathrm{N} / \mathrm{A}$ & JX438539 & $\mathrm{N} / \mathrm{A}$ \\
\hline C. notastroma & K20 & JX438629 & $\mathrm{N} / \mathrm{A}$ & $\mathrm{N} / \mathrm{A}$ & $\mathrm{N} / \mathrm{A}$ & JX438541 & $\mathrm{N} / \mathrm{A}$ \\
\hline C. olivacea & CFCC 53175 & MK673062 & MK673092 & MK673032 & MK673003 & $\mathrm{N} / \mathrm{A}$ & MK672978 \\
\hline C. olivacea & CFCC 53176 & MK673068 & MK673098 & MK673038 & MK673008 & MK672955 & MK672984 \\
\hline C. palm & CXY 1276 & JN402990 & $\mathrm{N} / \mathrm{A}$ & N/A & N/A & KJ781296 & N/A \\
\hline C.palm & CXY 1280 & JN411939 & $\mathrm{N} / \mathrm{A}$ & $\mathrm{N} / \mathrm{A}$ & $\mathrm{N} / \mathrm{A}$ & KJ781297 & $\mathrm{N} / \mathrm{A}$ \\
\hline C. parapersoonii & $\mathrm{T} 28.1$ & AF191181 & $\mathrm{N} / \mathrm{A}$ & $\mathrm{N} / \mathrm{A}$ & $\mathrm{N} / \mathrm{A}$ & JX438545 & $\mathrm{N} / \mathrm{A}$ \\
\hline C. paratranslucens & $\begin{array}{l}\text { MFLUCC } \\
15-0506\end{array}$ & KY417741 & KY417775 & KY417707 & KY417809 & $\mathrm{N} / \mathrm{A}$ & $\mathrm{N} / \mathrm{A}$ \\
\hline C. paratranslucens & $\begin{array}{l}\text { MFLUCC } \\
16-0627\end{array}$ & KY417742 & KY417776 & KY417708 & KY417810 & $\mathrm{N} / \mathrm{A}$ & $\mathrm{N} / \mathrm{A}$ \\
\hline C. pavettae & CBS 145562 & MK876386 & MK876427 & MK876457 & MK876483 & MK876497 & MK876503 \\
\hline C. phitsanulokensis & $\begin{array}{l}\text { MFLUCC } \\
21-0046\end{array}$ & MZ356517 & MZ356521 & MZ451160 & MZ451168 & MZ451164 & MZ451172 \\
\hline C. pingbianensis & $\begin{array}{l}\text { MFLUCC } \\
18-1204\end{array}$ & MK912135 & MK571763 & MN685817 & MN685826 & $\mathrm{N} / \mathrm{A}$ & $\mathrm{N} / \mathrm{A}$ \\
\hline C. pini & CBS 197.42 & AY347332 & $\mathrm{N} / \mathrm{A}$ & $\mathrm{N} / \mathrm{A}$ & $\mathrm{N} / \mathrm{A}$ & $\mathrm{N} / \mathrm{A}$ & $\mathrm{N} / \mathrm{A}$ \\
\hline C. pini & CBS 224.52 & AY347316 & $\mathrm{N} / \mathrm{A}$ & N/A & $\mathrm{N} / \mathrm{A}$ & $\mathrm{N} / \mathrm{A}$ & $\mathrm{N} / \mathrm{A}$ \\
\hline C.pini & СРC 28408 & KY051994 & KX965452 & KX964872 & KX965591 & $\mathrm{N} / \mathrm{A}$ & KX965041 \\
\hline C. platycladi & CFCC 50504 & MH933645 & МH933679 & MH933552 & MH933610 & MH933516 & MH933581 \\
\hline C. platycladi & CFCC 50506 & MH933647 & MH933681 & MH933554 & MH933612 & MH933518 & MH933583 \\
\hline C. plurivora & CBS 144239 & MG971861 & $\mathrm{N} / \mathrm{A}$ & MG972010 & $\mathrm{N} / \mathrm{A}$ & MG971572 & $\mathrm{N} / \mathrm{A}$ \\
\hline C. predappioensis & MFLU 17-0327 & MH253451 & MH253452 & MH253449 & MH253450 & N/A & $\mathrm{N} / \mathrm{A}$ \\
\hline C. predappioensis & $\begin{array}{l}\text { MFLUCC } \\
17-2458\end{array}$ & MG873484 & MG873480 & $\mathrm{N} / \mathrm{A}$ & $\mathrm{N} / \mathrm{A}$ & $\mathrm{N} / \mathrm{A}$ & $\mathrm{N} / \mathrm{A}$ \\
\hline C. pruinosa & CBS 201.42 & DQ243801 & $\mathrm{N} / \mathrm{A}$ & $\mathrm{N} / \mathrm{A}$ & $\mathrm{N} / \mathrm{A}$ & $\mathrm{N} / \mathrm{A}$ & N/A \\
\hline C. pruinosa & CBS 199.42 & KY051911 & KX965369 & KX964795 & $\mathrm{N} / \mathrm{A}$ & KX965159 & KX964968 \\
\hline C. pubescentis & $\begin{array}{l}\text { MFLUCC } \\
18-1201\end{array}$ & МK912130 & MK571758 & MN685812 & MN685821 & $\mathrm{N} / \mathrm{A}$ & $\mathrm{N} / \mathrm{A}$ \\
\hline C. punicae & CBS 144244 & MG971943 & $\mathrm{N} / \mathrm{A}$ & MG972091 & $\mathrm{N} / \mathrm{A}$ & MG971654 & $\mathrm{N} / \mathrm{A}$ \\
\hline C. punicae & 7C-11 & MG971942 & N/A & N/A & $\mathrm{N} / \mathrm{A}$ & MG971653 & $\mathrm{N} / \mathrm{A}$ \\
\hline C. quercicola & MFLU 17-0881 & MF190128 & MF190074 & $\mathrm{N} / \mathrm{A}$ & $\mathrm{N} / \mathrm{A}$ & $\mathrm{N} / \mathrm{A}$ & $\mathrm{N} / \mathrm{A}$ \\
\hline C. quercicola & $\begin{array}{l}\text { MFLUCC } \\
14-0867\end{array}$ & MF190129 & MF190073 & $\mathrm{N} / \mathrm{A}$ & $\mathrm{N} / \mathrm{A}$ & $\mathrm{N} / \mathrm{A}$ & $\mathrm{N} / \mathrm{A}$ \\
\hline C. rhizophorae & MUCC302 & EU301057 & $\mathrm{N} / \mathrm{A}$ & $\mathrm{N} / \mathrm{A}$ & $\mathrm{N} / \mathrm{A}$ & $\mathrm{N} / \mathrm{A}$ & $\mathrm{N} / \mathrm{A}$ \\
\hline C. rhizophorae & M225 & KR056292 & $\mathrm{N} / \mathrm{A}$ & $\mathrm{N} / \mathrm{A}$ & $\mathrm{N} / \mathrm{A}$ & $\mathrm{N} / \mathrm{A}$ & $\mathrm{N} / \mathrm{A}$ \\
\hline C. rhizophorae & ATCC 38475 & DQ996040 & $\mathrm{N} / \mathrm{A}$ & $\mathrm{N} / \mathrm{A}$ & $\mathrm{N} / \mathrm{A}$ & $\mathrm{N} / \mathrm{A}$ & $\mathrm{N} / \mathrm{A}$ \\
\hline C. rhizophorae & CBS 116861 & $\mathrm{~N} / \mathrm{A}$ & KX965296 & KX964735 & $\mathrm{N} / \mathrm{A}$ & $\mathrm{N} / \mathrm{A}$ & $\mathrm{N} / \mathrm{A}$ \\
\hline C. rosae & MFLU 17-0885 & MF190131 & MF190076 & $\mathrm{N} / \mathrm{A}$ & $\mathrm{N} / \mathrm{A}$ & $\mathrm{N} / \mathrm{A}$ & $\mathrm{N} / \mathrm{A}$ \\
\hline C. rusanovii & $\begin{array}{l}\text { MFLUCC } \\
15-0853\end{array}$ & KY417743 & KY417777 & KY417709 & KY417811 & $\mathrm{N} / \mathrm{A}$ & $\mathrm{N} / \mathrm{A}$ \\
\hline C. rusanovii & $\begin{array}{l}\text { MFLUCC } \\
\text { 15-0854 }\end{array}$ & KY417744 & KY417778 & KY417710 & KY417812 & $\mathrm{N} / \mathrm{A}$ & $\mathrm{N} / \mathrm{A}$ \\
\hline
\end{tabular}


Table 2. Cont.

\begin{tabular}{|c|c|c|c|c|c|c|c|}
\hline \multirow{2}{*}{ Species Name } & \multirow{2}{*}{$\begin{array}{c}\text { Culture } \\
\text { Accession No. }\end{array}$} & \multicolumn{6}{|c|}{ Genbank Accession No. } \\
\hline & & ITS & LSU & ACT & RPB2 & TEF1- $\alpha$ & TUB2 \\
\hline C. shoreae & $\begin{array}{l}\text { MFLUCC } \\
21-0047\end{array}$ & MZ356515 & MZ356519 & MZ451158 & MZ451166 & MZ451162 & MZ451170 \\
\hline C. shoreae & $\begin{array}{l}\text { MFLUCC } \\
21-0048\end{array}$ & MZ356516 & MZ356520 & MZ451159 & MZ451167 & MZ451163 & MZ451171 \\
\hline C. sorbi & $\begin{array}{l}\text { MFLUCC } \\
16-0631\end{array}$ & KY417752 & KY417786 & KY417718 & KY417820 & $\mathrm{N} / \mathrm{A}$ & $\mathrm{N} / \mathrm{A}$ \\
\hline C. sorbicola & $\begin{array}{l}\text { MFLUCC } \\
16-0584\end{array}$ & KY417755 & KY417789 & KY417721 & KY417823 & $\mathrm{N} / \mathrm{A}$ & $\mathrm{N} / \mathrm{A}$ \\
\hline C. sorbicola & $\begin{array}{l}\text { MFLUCC } \\
16-0633\end{array}$ & KY417758 & KY417792 & KY417724 & KY417826 & $\mathrm{N} / \mathrm{A}$ & $\mathrm{N} / \mathrm{A}$ \\
\hline C. tamaricicola & CFCC 50507 & МH933651 & MH933686 & МH933559 & МH933616 & MH933525 & MH933587 \\
\hline C. tamaricicola & CFCC 50508 & MH933652 & MH933687 & MH933560 & MH933617 & MH933523 & MH933588 \\
\hline C. thailandica & $\begin{array}{l}\text { MFLUCC } \\
17-0262\end{array}$ & MG975776 & MH253455 & MH253459 & MH253463 & $\mathrm{N} / \mathrm{A}$ & $\mathrm{N} / \mathrm{A}$ \\
\hline C. thailandica & $\begin{array}{l}\text { MFLUCC } \\
17-0263\end{array}$ & MG975777 & MH253456 & MH253460 & MH253464 & $\mathrm{N} / \mathrm{A}$ & $\mathrm{N} / \mathrm{A}$ \\
\hline C. translucens & CXY 1351 & KM034874 & N/A & $\mathrm{N} / \mathrm{A}$ & N/A & N/A & KM034895 \\
\hline C. valsoidea & CBS 117003 & KY051832 & KX965298 & $\mathrm{N} / \mathrm{A}$ & KX965494 & KX965097 & N/A \\
\hline C. variostromatica & CMW 6766 & AY347366 & N/A & $\mathrm{N} / \mathrm{A}$ & $\mathrm{N} / \mathrm{A}$ & $\mathrm{N} / \mathrm{A}$ & $\mathrm{N} / \mathrm{A}$ \\
\hline C. variostromatica & PPRI5297 & AF260264 & N/A & N/A & $\mathrm{N} / \mathrm{A}$ & $\mathrm{N} / \mathrm{A}$ & N/A \\
\hline C. variostromatica & CBS 116858 & KY051828 & KX965293 & KX964732 & $\mathrm{N} / \mathrm{A}$ & $\mathrm{N} / \mathrm{A}$ & KX964921 \\
\hline C. vinacea & CBS 141585 & KX256256 & $\mathrm{N} / \mathrm{A}$ & $\mathrm{N} / \mathrm{A}$ & $\mathrm{N} / \mathrm{A}$ & KX256277 & KX256235 \\
\hline C. viridistroma & CBS 202.36 & MN172408 & MN172388 & $\mathrm{N} / \mathrm{A}$ & $\mathrm{N} / \mathrm{A}$ & MN271853 & $\mathrm{N} / \mathrm{A}$ \\
\hline C. viridistroma & CRY 1534 & AF452120 & N/A & N/A & N/A & $\mathrm{N} / \mathrm{A}$ & $\mathrm{N} / \mathrm{A}$ \\
\hline C. xinglongensis & CFCC 52458 & MK432622 & MK429892 & MK442946 & MK578082 & $\mathrm{N} / \mathrm{A}$ & $\mathrm{N} / \mathrm{A}$ \\
\hline C. xinglongensis & CFCC 52459 & MK432623 & MK429893 & MK442947 & MK578083 & $\mathrm{N} / \mathrm{A}$ & $\mathrm{N} / \mathrm{A}$ \\
\hline C. xylocarpi & $\begin{array}{l}\text { MFLUCC } \\
\text { 17-0251 }\end{array}$ & MG975775 & MH253454 & MH253458 & MH253462 & $\mathrm{N} / \mathrm{A}$ & $\mathrm{N} / \mathrm{A}$ \\
\hline C. xylocarpi & 111_03_01 & MT507847 & $\mathrm{N} / \mathrm{A}$ & N/A & $\mathrm{N} / \mathrm{A}$ & N/A & N/A \\
\hline Diaporthe eres & CBS 138594 & KJ210529 & $\mathrm{N} / \mathrm{A}$ & KJ420760 & $\mathrm{N} / \mathrm{A}$ & KJ210550 & KJ420799 \\
\hline D. vaccinii & CBS 160.32 & КС 343228 & $\mathrm{~N} / \mathrm{A}$ & JQ807297 & $\mathrm{N} / \mathrm{A}$ & КС343954 & КС344196 \\
\hline
\end{tabular}

Maximum likelihood (ML) analysis was performed by RAxML-HPC2 (v.8.2.12) on XSEDE implemented in the CIPRES Science Gateway web server (http:/ / www.phylo.org accessed on 1 August 2021; [37] using 1000 rapid bootstrap replicates and the GTR + GAMMA + I substitution model. Maximum parsimony (MP) analysis was generated by PAUP (Phylogenetic Analysis Using Parsimony) v.4.0b10 [38] using the heuristic search option and 1000 random sequence additions. The branch-swapping was analysed using tree-bisection reconnection (TBR) algorithm. Maxtrees was set up at 1000 and all characters were unordered and of equal weight. The branches of zero length were collapsed and gaps were treated as missing data. All multiple and equally parsimonious trees were saved. The stability of the most parsimonious tree was evaluated by a bootstrap analysis with 1000 replicates, each with 100 replicates of random stepwise addition of taxa. The descriptive parsimonious tree, Tree Length [TL], Consistency Index [CI], Retention Index [RI], Relative Consistency Index [RC] and Homoplasy Index [HI] were calculated.

The evolutionary models for Bayesian inference (BI) analysis were determined for each locus using MrModeltest v.2.3 [39]. GTR + I + G was selected as the best-fitting model for LSU, RPB2, TEF- $\alpha$ and TUB2 datasets, SYM + I + G for the ITS dataset and HKY $+\mathrm{I}+\mathrm{G}$ for the ACT dataset. BI analysis was conducted by Markov chain Monte Carlo sampling (BMCMC) to assess posterior probabilities (PP) [40,41] using MrBayes v3.1.2 [42]. Six simultaneous Markov chains were run for random trees for 10,000,000 generations, and trees were sampled every 1000th generation. The effective sampling sites (ESS) of initial trees were checked using the Tracer v. 1.6 [43]. The first 10\% of generated trees were discarded, and the remaining trees were used to calculate posterior probabilities (PP) 
in the majority rule consensus tree (the standard deviation of split frequency lower than 0.01). Bootstrap support values for ML and MP equal to or greater than $60 \%$ and Bayesian posterior probabilities (PP) equal to or greater than 0.95 were given above the nodes in the phylogenetic tree (Figure 1).

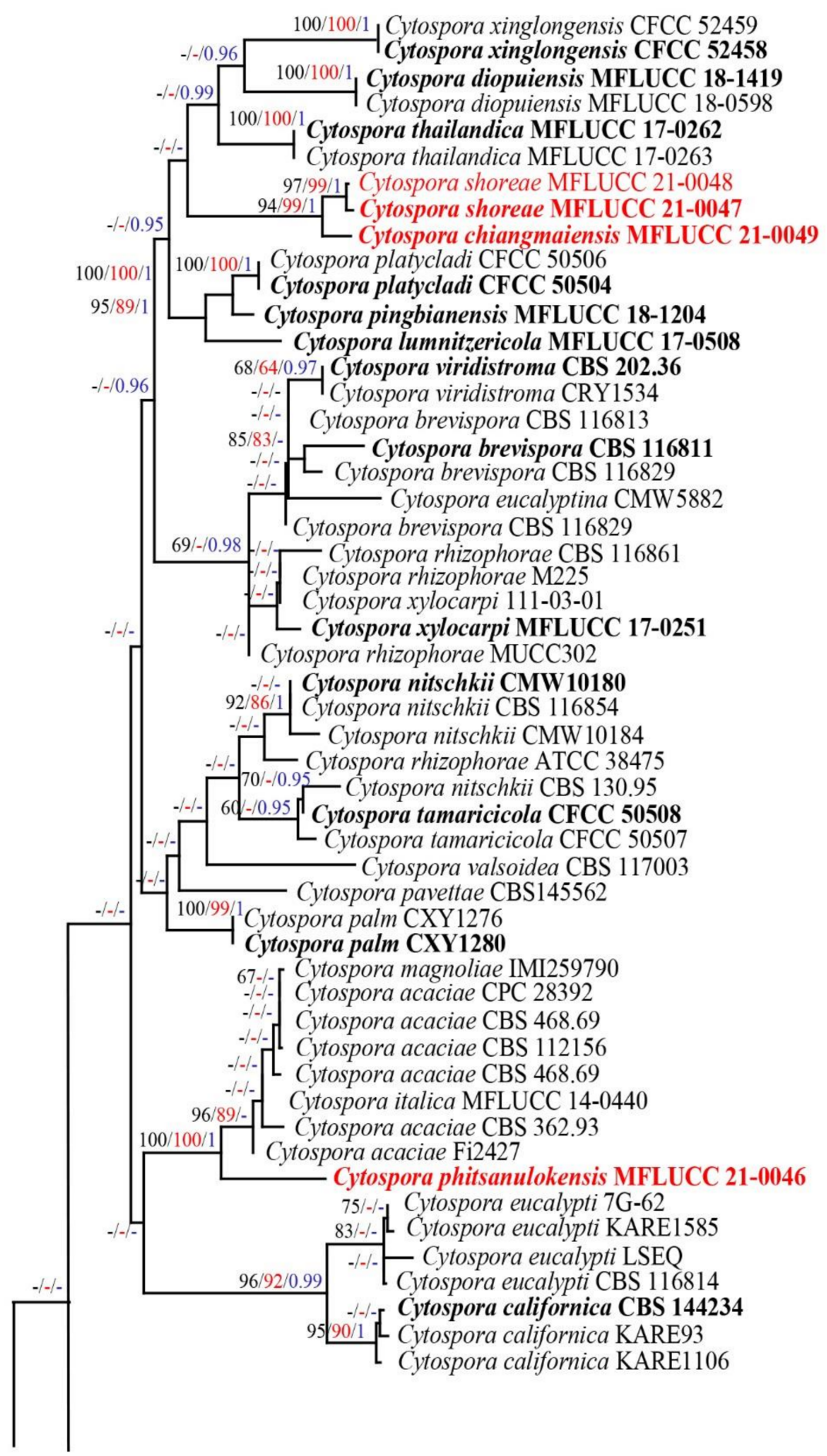

Figure 1. Cont. 


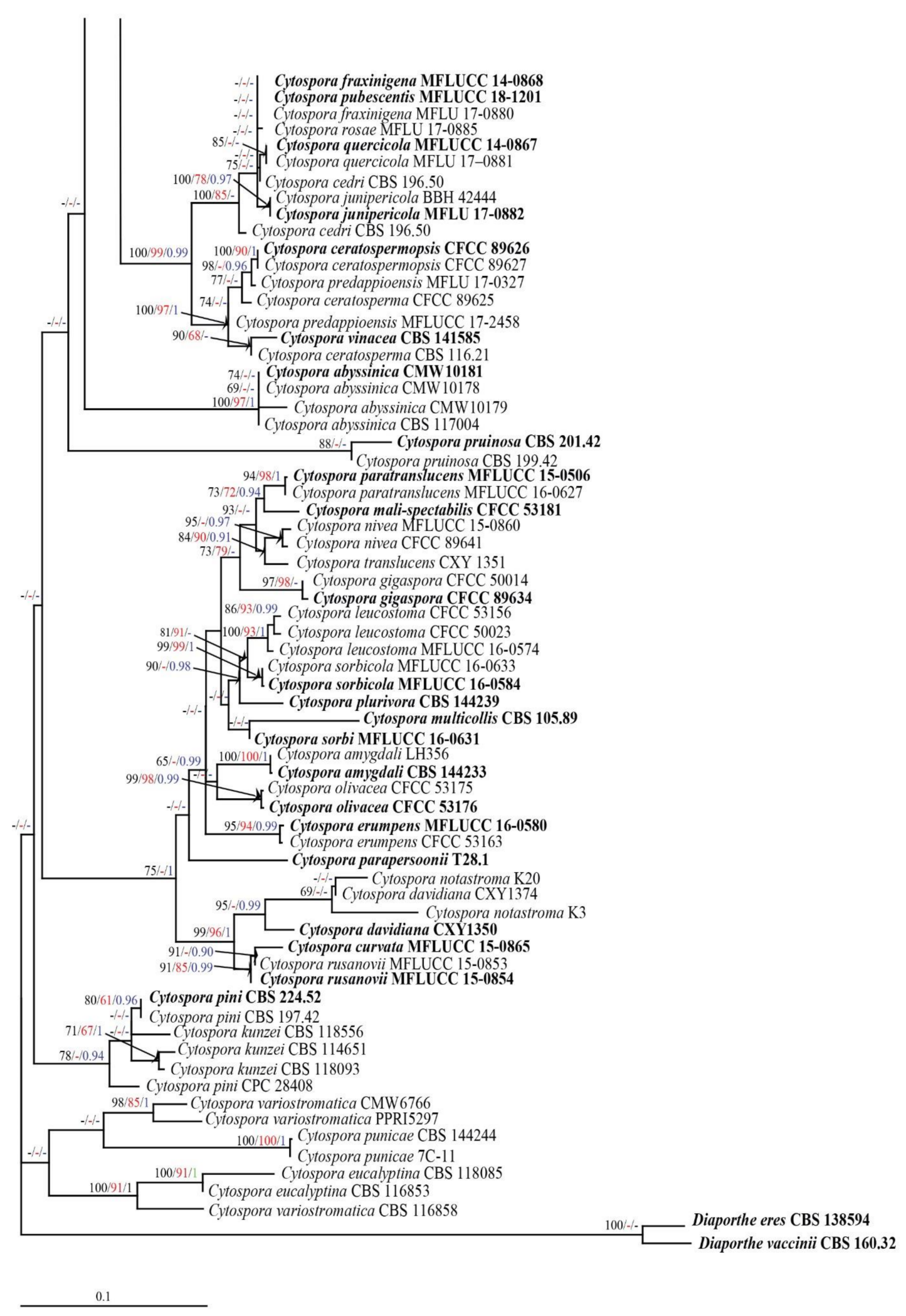

Figure 1. Phylogenetic tree obtained from RAxML analyses of a combined ITS, LSU, ACT, RPB2, TEF1- $\alpha$ and TUB2 sequence dataset. Bootstrap support values for ML, MP equal to or greater than $60 \%$ and PP equal to greater than 0.95 are indicated at the nodes as ML/MP/PP. The ex-type strains are in bold and the new isolates of this study are in red. The tree is rooted to Diaporthe eres (CBS138594) and D. vaccinii (CBS16032). 
Phylograms were visualized by FigTree v1.4.0 program [44] and reorganized by Microsoft PowerPoint 2013 (Microsoft Inc., Redmond, WA, USA) and Adobe Photoshop CS6 software (Adobe Systems, San Jose, CA, USA). The generated sequences of the new taxa were registered for the GenBank accession numbers. The final alignment and phylogram were submitted in TreeBASE (http://purl.org/phylo/treebase/phylows/study/TB2:S288 04 accessed on 1 August 2021).

\subsection{Genealogical Concordance Phylogenetic Species Recognition Analysis}

New species and phylogenetically related species were analyzed using the Genealogical Concordance Phylogenetic Species Recognition (GCPSR) model by conducting a pairwise homoplasy index (PHI) test as described by Bruen et al. [45] and Quaedvlieg et al. [46]. The PHI test was conducted in SplitsTree4 $[47,48]$ to examine the recombination level within phylogenetically closely related species using a six-locus concatenated dataset (ITS, LSU, ACT, RPB2, TEF1- $\alpha$ and TUB2). The significant recombination in the dataset was indicated by PHI value below $0.05\left(\Phi_{\mathrm{w}}<0.05\right)$. The results were visualized by generating a split graph, using both the LogDet transformation and split decomposition options.

\subsection{Preliminary Screening of Antagonistic Activity against Fungal Pathogens}

The fungal pathogens, Colletotrichum artocarpicola (MFLUCC 18-1167), Co. fructicola (MFLUCC 18-1160), Co. siamense (MFLUCC 18-1162), Co. viniferum (MFLUCC 18-1179) and Fusarium sambucinum (MFLUCC 17-1056) were obtained from MFLUCC and used for the antagonistic activity test. Fungal isolates were screened using in vitro dual culture assays for their ability to suppress the mycelial growth of fungal pathogens. An antagonism test was performed with 10-day-old cultures of pathogens and new strains. Fresh cultures (pathogens and our strains) 10 days after incubation were used for the antagonism test. A fungal pathogen disc $(5 \mathrm{~mm})$ was placed $3 \mathrm{~cm}$ from the margin of the PDA plate $(9 \mathrm{~cm}$ in diam.). An antagonist fungus disc $(5 \mathrm{~mm})$ was also placed in a similar manner but on the direct opposite of the pathogen disc. The plate was incubated at room temperature $\left(28^{\circ} \mathrm{C}\right)$ for 10 days. Plates inoculated with a fungal pathogen in the absence of an antagonistic fungus were used as negative controls. The assay was replicated three times. Observations were carried out for the 3rd, 5th, 7th and 10th days. Clear inhibition zone was recorded and the percentage inhibition in mycelial growth was calculated using the following formula [49]: I\% = [(R1-R2)/R1] $\times 100$, where $\mathrm{I} \%=$ the percentage inhibition, $\mathrm{R} 1=$ the radial growth of test pathogen in a control plate and $\mathrm{R} 2=$ the radial growth of test pathogen in the direction of antagonistic fungus. Data were statistically analyzed with ANOVA using SPSS version 22 (SPSS, Inc., Chicago, IL, USA). Tukey's HSD test was used to determine the significant differences between treatments at $p \leq 0.05$.

\section{Results}

\subsection{Phylogenetic Analyses}

The concatenated sequence dataset of ITS, LSU, ACT, RPB2, TEF1- $\alpha$ and TUB2 gene regions comprised 120 strains of Cytospora and two outgroup taxa, Diaporthe eres (CBS 138594) and D. vaccinia (CBS 160.32). The dataset contained 3339 characters according to the order of ITS: 1-416, LSU: 417-1199, ACT: 1200-1429, RPB2: 1430-2027, TEF- $\alpha$ : 2028-2624, $\beta$-TUB: $2625-3339$. The tree topologies of combined sequence data obtained from ML, MP and $\mathrm{BI}$ analyses were not significantly different.

The RAxML analysis of the combined dataset yielded a best scoring tree with a final ML optimization likelihood value of -24983.102448 (Figure 1). The aligned sequence matrix comprises 1399 distinct alignment patterns, with $55.00 \%$ of undetermined characters or gaps. Estimated base frequencies were as follows: $A=0.239305, C=0.269679, G=0.260287$, $\mathrm{T}=0.230728$; substitution rates $\mathrm{AC}=1.822160, \mathrm{AG}=4.370331, \mathrm{AT}=1.771054, \mathrm{CG}=1.304544$, $\mathrm{CT}=8.574513, \mathrm{GT}=1.000000$; gamma distribution shape parameter $\alpha=0.219288$. The maximum parsimonious dataset consisted of 3354 characters, of which 2127 were constant, 1052 were parsimony-informative and 175 were parsimony-uninformative. The descriptive 
statistics of the most parsimony tree was $\mathrm{TL}=4294, \mathrm{CI}=0.468, \mathrm{RI}=0.780, \mathrm{RC}=0.365$, $\mathrm{HI}=0.532$.

In the phylogenetic analyses (Figure 1), two new species, Cytospora chiangmaiensis (MFLUCC 21-0049) and Cytospora shoreae (MFLUCC 21-0047, MFLUCC 21-0048), clustered in a monophyletic lineage but well separated branch with strong bootstrap support (94\% ML/99\% MP/1.00 PP). Another new species, Cytospora phitsanulokensis (MFLUCC 21-0046), formed an independent branch adjacent to C. acaciae, C. magnoliae and C. italica with high bootstrap support (100\% ML, 100\% MP, 1.00 PP; Figure 1).

A pairwise homoplasy index (PHI) test revealed no significant recombination event between Cytospora phitsanulokensis and the closely related taxa, C. acaciae, C. italica and C. magnoliae (Figure 2). There was also no significant recombination among Cytospora chiangmaiensis, C. shoreae, C. thailandica, C. diopuiensis and C. xinglongensis (Figure 2). This evidence supports that they are different species. The significant recombination between two strains of Cytospora shoreae (MFLUCC 21-0047 and MFLUCC 21-0048) indicate that they are conspecific (Figure 3).

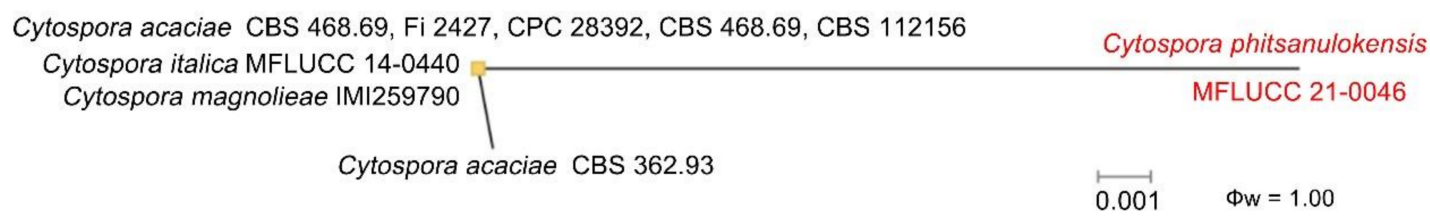

Figure 2. Results of the pairwise homoplasy index (PHI) test of Cytospora phitsanulokensis and closely related species using both LogDet transformation and splits decomposition. PHI test results $\left(\Phi_{\mathrm{W}}\right)<0.05$ indicate significant recombination within the dataset. New species described in this study are indicated in red.

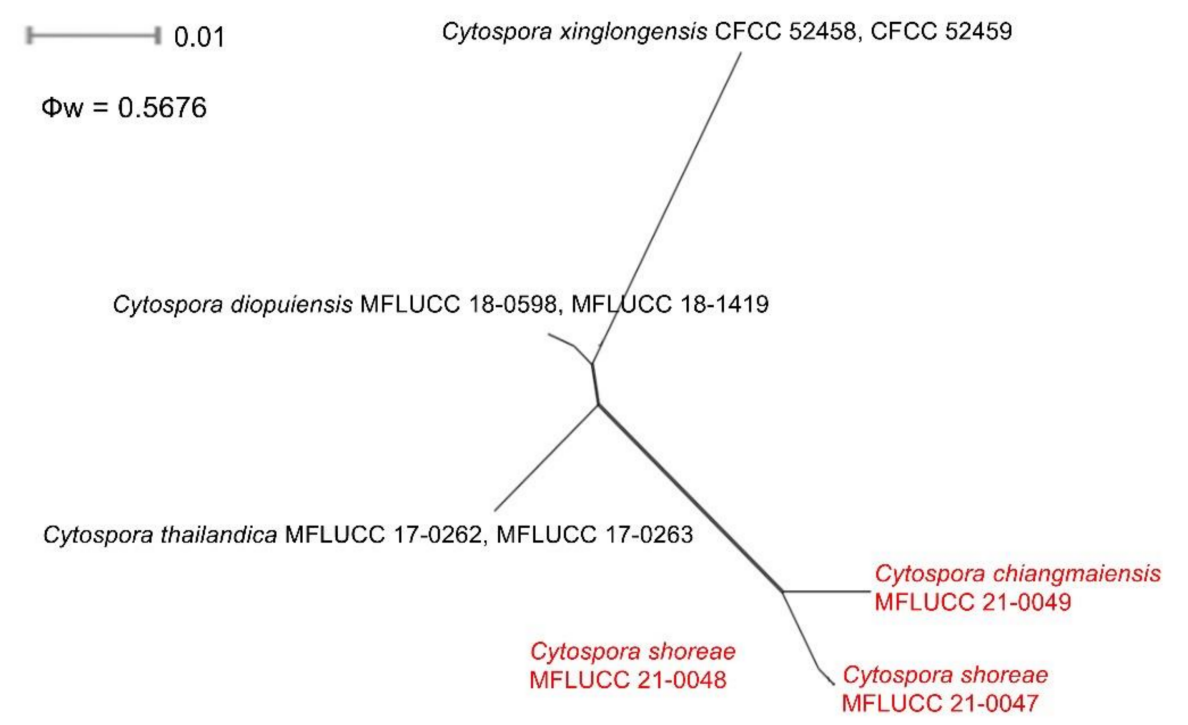

Figure 3. Results of the pairwise homoplasy index (PHI) test of Cytospora chiangmaiensis and C. shoreae and closely related species using both LogDet transformation and splits decomposition. PHI test results $\left(\Phi_{\mathrm{w}}\right)<0.05$ indicate significant recombination within the dataset. New species described in this study are indicated in red.

\subsection{Taxonomy}

3.2.1. Cytospora chiangmaiensis Monkai and K.D. Hyde, sp. nov.

Index Fungorum number: IF558524; Facesoffungi number: FoF 09935; Figure 4. 

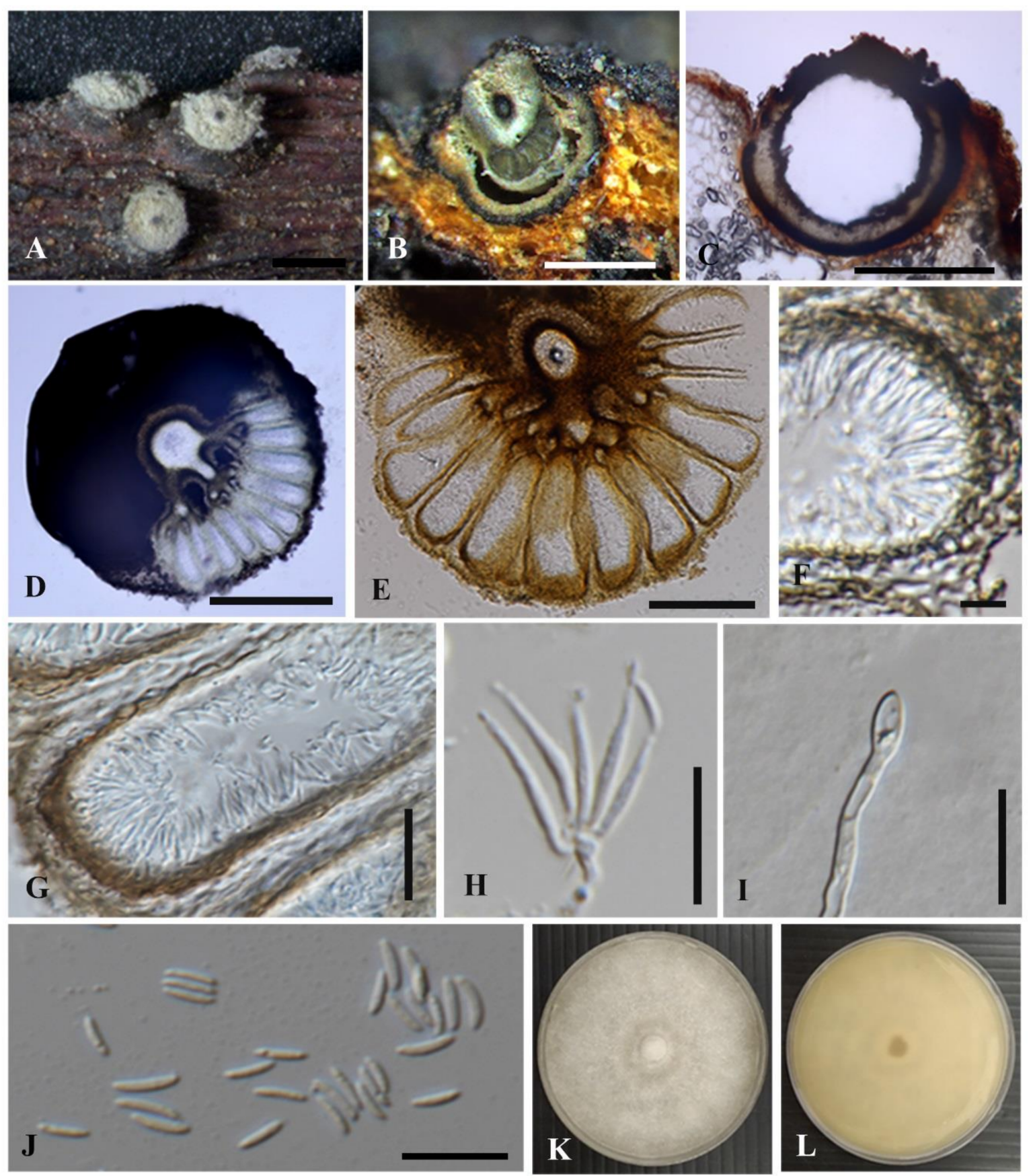

Figure 4. Cytospora chiangmaiensis (MFLU 21-0048, holotype). (A) Conidiomata on host substrate. (B-D) Longitudinal sections through conidioma. (E) Conidiomata with ostiole and the arrangement of locules. (F,G) Peridium. (H) Conidiophore and conidiogenous cells with attached conidia. (I) Germinating conidia. (J) Conidia. (K,L) Colony on PDA plate $(9 \mathrm{~cm}$ diam.) $((\mathbf{K})$ from above, $(\mathbf{L})$ from reverse). Scale bars: $(\mathbf{A}-\mathbf{C})=500 \mu \mathrm{m},(\mathbf{D}, \mathbf{E})=100 \mu \mathrm{m},(\mathbf{G})=50 \mu \mathrm{m},(\mathbf{F}, \mathbf{H}-\mathbf{J})=10 \mu \mathrm{m}$.

Etymology: Name reflects the locality, Chiang Mai Province, Thailand, where the holotype was collected.

Holotype: MFLU 21-0048

Saprobic on decaying leaves (vein and petioles) of Shorea sp. Sexual morph: Undetermined. Asexual morph: Conidiomata 650-800 $\mu \mathrm{m}$ diam., pycnidial, solitary, semiimmersed to erumpent, circular to ovoid, multi-loculate. Conceptacle black. Ectostromatic disc 170-230 $\mu \mathrm{m}$ diam., brown to dark brown, circular to ovoid, one ostiole per disc. Ostioles 55-65 $\mu \mathrm{m}$ diam., conspicuous, circular, dark brown, at the same level as the disc surface. Peridium comprising few layers of cells, textura globosa, dark brown to black. Locules 
numerous, regularly arranged with a column, independent wall. Conidiophores hyaline, unbranched or occasionally branched at the bases, formed from the innermost layer of pycnidial wall, embedded in a gelatinous layer. Conidiogenous cells 8.5-12.2 $\times 1-1.7 \mu \mathrm{m}$ $(\bar{x}=10.4 \times 1.3 \mu \mathrm{m}, n=20)$, enteroblastic, phialidic, sub-cylindrical to cylindrical, tapering towards apices. Conidia 5-7 $\times 0.9-1.7 \mu \mathrm{m}(\bar{x}=6 \times 1.2 \mu \mathrm{m}, n=30)$, hyaline, unicellular, elongate-allantoid, guttulate, smooth, thin-walled.

Culture characteristics: Conidia germinating on PDA within $24 \mathrm{~h}$ germ tubes produced from one pole. Colonies on PDA reached at $5 \mathrm{~cm}$ diam. after 7 days at $28^{\circ} \mathrm{C}$, irregular in shape, surface slightly rough, effuse, slightly raised, with undulate margin, medium dense, white to cream, in reverse pale yellowish to white.

Material examined: THAILAND, Chiang Mai, Doi Lor district, Yang Kram, on decaying leaves of Shorea sp. (Dipterocarpaceae), 15 October 2019, J. Monkai, CH1-2 (MFLU 21-0048, holotype), ex-type living culture, MFLUCC 21-0049.

Notes: Cytospora chiangmaiensis formed an independent lineage sister to Cytospora shoreae with $94 \% \mathrm{ML} / 99 \% \mathrm{MP} / 1.00$ PP bootstrap support (Figure 1). Based on phylogenetic analyses, Cytospora chiangmaiensis grouped in the same clade with $C$. diopuiensis, C. lumnitzericola, C. pingbianensis, C. platycladi C. shoreae, C. thailandica and C. xinglongensis (Figure 1). Cytospora diopuiensis and C. pingbianensis are only known from their sexual morph [2], and our strain was found as an asexual morph. Thus, we are unable to compare the morphological characters of those species. However, Cytospora lumnitzericola can be distinguished from $C$. chiangmaiensis by its shorter conidia [5] (Table 4). Cytospora platycladi is similar to C. chiangmaiensis in having multi-loculate conidiomata with central ostiole but it has smaller conidiomata and shorter conidia [1] (Table 4). Cytospora thailandica differs from C. chiangmaiensis in having larger conidiomata with an ostiolar neck, shorter conidiogenous cell and shorter conidia [5] (Table 4). Cytospora xinglongensis differs from C. chiangmaiensis in having undivided locules, inconspicuous ostioles and shorter conidiogenous cells and longer conidia [16] (Table 4).

\subsubsection{Cytospora shoreae Monkai and K.D. Hyde, sp. nov.}

Index Fungorum number: IF558525; Facesoffungi number: FoF 09936; Figure 5.

Etymology: The specific epithet shoreae refers to host plant genus Shorea, on which the fungus was first collected.

Holotype: MFLU 21-0049

Saprobic on decaying leaves (vein and petioles) of Shorea sp. Sexual morph: Undetermined. Asexual morph: Conidiomata 400-1000 $\mu \mathrm{m}$ diam., pycnidial, semi-immersed in host tissue, solitary, erumpent, flask shaped to ovoid, multi-loculate. Conceptacle black. Ectostromatic disc 120-580 $\mu \mathrm{m}$ diam., brown to dark brown, circular to ovoid, one ostiole per disc. Ostioles 19-52 $\mu \mathrm{m}$ diam., conspicuous, circular, dark brown, at the same level as the disc surface. Peridium comprising few layers of cells, textura globosa, dark brown to black. Locules numerous, regularly arranged with a column, independent wall. Conidiophores hyaline, unbranched or occasionally branched at the bases, formed from the innermost layer of pycnidial wall, embedded in a gelatinous layer. Conidiogenous cells 6.7-11.8 $\times 1-1.9 \mu \mathrm{m}$ $(\bar{x}=10 \times 1.4 \mu \mathrm{m}, n=10)$, enteroblastic, phialidic, subcylindrical to cylindrical, tapering towards apices. Conidia 5-7 $\times 1-1.6 \mu \mathrm{m}(\bar{x}=6 \times 1.3 \mu \mathrm{m}, n=30)$, hyaline, unicellular, elongate-allantoid, guttulate, smooth, thin-walled.

Culture characteristics: Conidia germinating on PDA within $24 \mathrm{~h}$ germ tubes produced from both poles. Colonies on PDA reached at $6 \mathrm{~cm}$ diam. after 7 days at $28^{\circ} \mathrm{C}$, irregular in shape, surface slightly rough, effuse, slightly raised, with undulate margin, medium dense, pale brown to white, in reverse pale yellowish to white. 

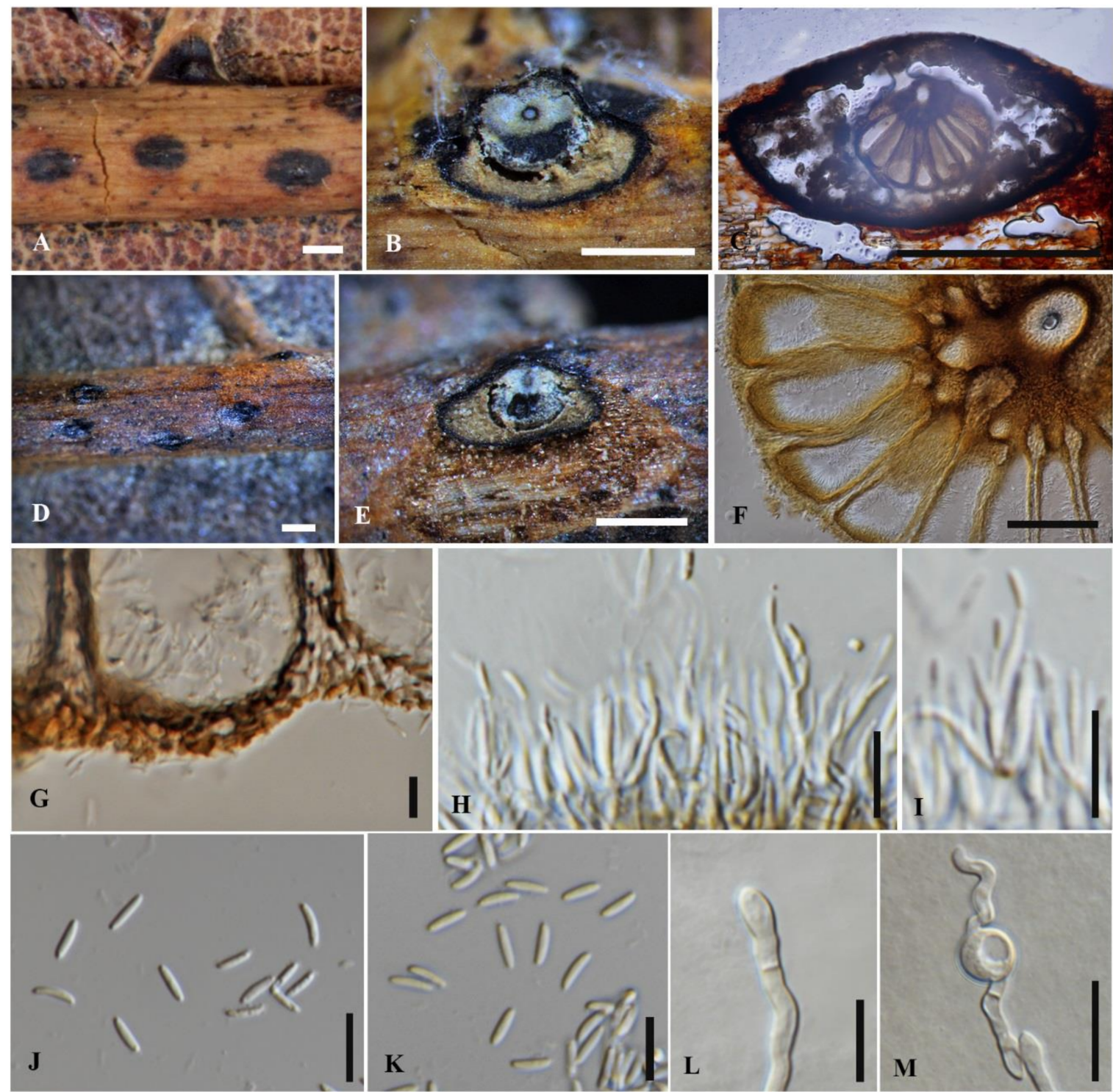

Ho
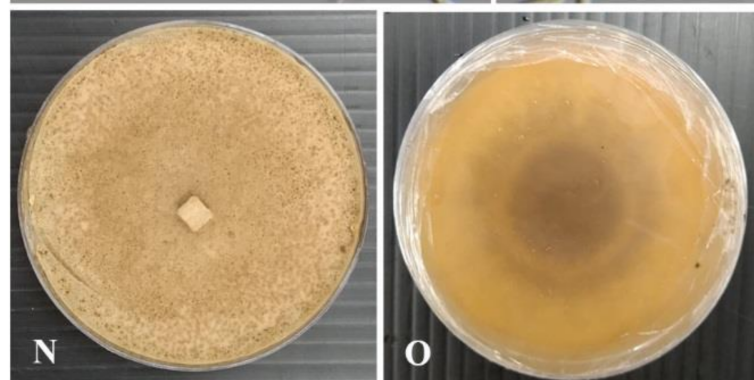

$\mathbf{L}$
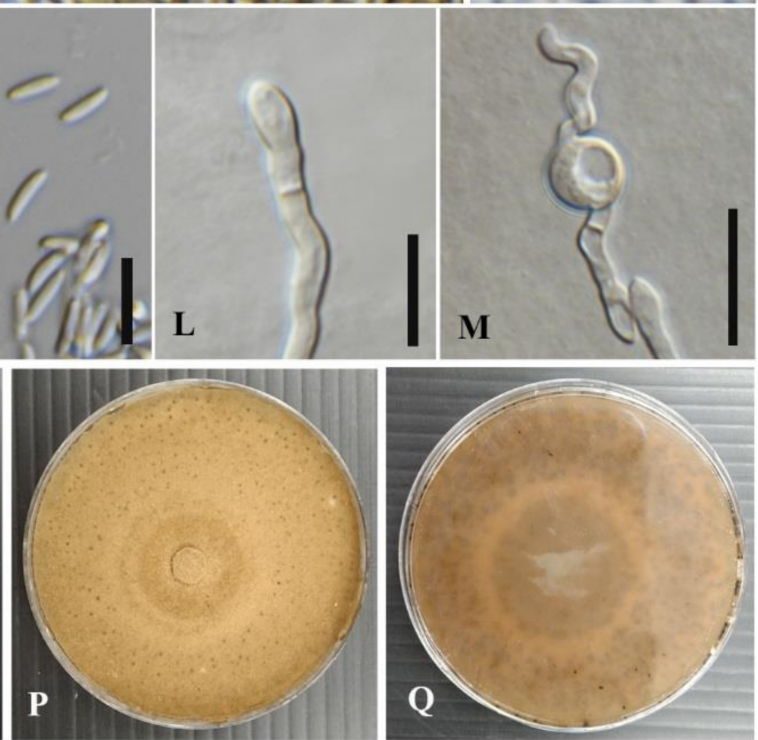

Figure 5. Cytospora shoreae (MFLU 21-0049, holotype (C-E,G,H,J,L,N,O) and MFLU 21-0050, paratype (A,B,F,I,K,M,P,G)). (A,D) Conidiomata on host substrate. (B,C,E,F) Longitudinal sections through conidioma. (G) Peridium. (H,I) Conidiophores and conidiogenous cells with attached conidia. (J,K) Conidia. (L,M) Germinating conidia. (N-Q) Colonies on PDA plate $(9 \mathrm{~cm}$ diam.) $((\mathbf{N}, \mathbf{P})$ from above, $(\mathbf{O}, \mathbf{Q})$ from reverse). Scale bars: $(\mathbf{A}-\mathbf{E})=500 \mu \mathrm{m},(\mathbf{F})=50 \mu \mathrm{m},(\mathbf{G}-\mathbf{M})=10 \mu \mathrm{m}$.

Material examined: THAILAND, Chiang Mai, Omkoi district, Yang Piang, on decaying leaves of Shorea sp. (Dipterocarpaceae), 16 October 2019, J. Monkai, CH2-2 (MFLU 21-0049, holotype), ex-type living culture, MFLUCC 21-0047; THAILAND, Chiang Mai, Omkoi district, Yang Piang, on unidentified decaying leaves, 16 October 2019, J. Monkai, CH2-3 (MFLU 21-0050, paratype), ex-paratype living culture MFLUCC 21-0048. 
Notes: Cytospora shoreae formed a sister clade to C. chiangmaiensis with $94 \% \mathrm{ML}$, 99\% MP and 1.00 PP statistical support (Figure 1). Cytospora chiangmaiensis and C. shoreae share similar morphology in the size and characteristics of conidiogenous cells and conidia. However, C. chiangmaiensis has circular shaped conidiomata. Cytospora shoreae has larger, flask-shaped conidiomata. The single gene comparison of ACT, RPB2, TEF1- $\alpha$ and TUB2 showed that there are significant nucleotide differences (more than 1.5\%) between Cytospora chiangmaiensis and two strains of C. shoreae (MFLUCC 21-0047 and MFLUCC 21-0048; Table 3) and this provides evidence that they are different species [50]. Cytospora shoreae differs from C. lumnitzericola and C. platycladi by its longer conidia [1,5] (Table 4). Cytospora shoreae is distinguished from $C$. thailandica by having longer conidiogenous cell and conidia [5] (Table 4). Cytospora shoreae is distinct from C. xinglongensis in having longer conidiogenous cells and shorter conidia [16] (Table 4). Cytospora shoreae, strains MFLUCC 21-0047 and MFLUCC 21-0048 showed similar characteristics of conidiomata, conidiogenous cells, conidia and culture characteristics, with no significant differences. They were also collected from the same site with the notes of different hosts, where the holotype was isolated from Shorea sp. and the paratype was isolated from unidentified host. The single gene comparison of ITS, LSU, ACT, RPB2, TEF1- $\alpha$ and TUB2 showed that there is no significant difference between two strains of Cytospora shoreae (MFLUCC 21-0047 and MFLUCC 210048; Table 3), and this confirms that these two strains are the same species [50].

Table 3. Nucleotide differences of Cytospora species discussed in this study.

\begin{tabular}{|c|c|c|c|c|c|c|c|}
\hline \multirow{2}{*}{ New Taxa/Strains } & \multirow{2}{*}{$\begin{array}{l}\text { New Taxa/Strains } \\
\text { Compared with }\end{array}$} & \multicolumn{6}{|c|}{$\begin{array}{l}\text { Number of Different Nucleotides/Number of All Nucleotides } \\
\text { (\% Base Pairs Difference) }\end{array}$} \\
\hline & & ITS & LSU & ACT & RPB2 & TEF1- $\alpha$ & TUB2 \\
\hline $\begin{array}{l}\text { Cytospora shoreae } \\
\text { (MFLUCC 21-0047) }\end{array}$ & $\begin{array}{c}\text { C. shoreae } \\
\text { (MFLUCC 21-0048) }\end{array}$ & $0 / 494(0 \%)$ & $0 / 510(0 \%)$ & $0 / 180(0 \%)$ & $\begin{array}{l}4 / 726 \\
(0.6 \%)\end{array}$ & 0/291 (0\%) & $\begin{array}{l}3 / 375 \\
(0.8 \%)\end{array}$ \\
\hline $\begin{array}{l}\text { C. chiangmaiensis } \\
\text { (MFLUCC 21-0049) }\end{array}$ & $\begin{array}{c}\text { C. shoreae } \\
\text { (MFLUCC 21-0047) }\end{array}$ & $0 / 494(0 \%)$ & $0 / 510(0 \%)$ & $\begin{array}{l}5 / 180 \\
(2.8 \%)\end{array}$ & $\begin{array}{l}13 / 726 \\
(1.8 \%)\end{array}$ & $\begin{array}{l}16 / 291 \\
(5.5 \%)\end{array}$ & $\begin{array}{l}13 / 375 \\
(3.5 \%)\end{array}$ \\
\hline $\begin{array}{l}\text { C. chiangmaiensis } \\
\text { (MFLUCC 21-0049) }\end{array}$ & $\begin{array}{c}\text { C. shoreae } \\
\text { (MFLUCC 21-0048) }\end{array}$ & 0/494 (0\%) & $0 / 510(0 \%)$ & $\begin{array}{l}5 / 180 \\
(2.8 \%)\end{array}$ & $\begin{array}{l}11 / 726 \\
(1.5 \%)\end{array}$ & $\begin{array}{l}16 / 291 \\
(5.5 \%)\end{array}$ & $\begin{array}{l}12 / 375 \\
(3.2 \%)\end{array}$ \\
\hline
\end{tabular}

Table 4. Synopsis of asexual morph of Cytospora species discussed in this study.

\begin{tabular}{lcccc}
\hline \multicolumn{1}{c}{ Species } & Conidiomata $(\mu \mathrm{m})$ & Conidiogenous Cell $(\boldsymbol{\mu m})$ & Conidia $(\mu \mathbf{m})$ & References \\
\hline Cytospora chiangmaiensis & $650-800$ & $8.5-12.2 \times 1-1.7$ & $5-7 \times 0.9-1.7$ & This study \\
C. italica & $580-730$ & $10-32 \times 0.7-2.4$ & $3.7-5.2 \times 1-1.3$ & {$[51]$} \\
C. lumnitzericola & Undetermined & $8-14 \times 0.6-1.6$ & $3.7-4.5 \times 1-1.5$ & {$[5]$} \\
C. platycladi & $210-330$ & $5-12 \times 1-1.5$ & $4-5.5 \times 1-1.5$ & {$[1]$} \\
C. shoreae & $400-1000$ & $6.7-11.8 \times 1-1.9$ & $5-7 \times 1-1.6$ & This study \\
C. thailandica & $400-1200$ & $3.3-9.1 \times 1-1.7$ & $3.3-4 \times 1-1.5$ & {$[5]$} \\
C. xinglongensis & Undetermined & $4.5-12 \times 1-1.5$ & $7.5-10.5 \times 1-1.5$ & {$[16]$} \\
\hline
\end{tabular}

3.2.3. Cytospora phitsanulokensis Monkai and K.D. Hyde, sp. nov. Index Fungorum number: IF558526; Facesoffungi number: FoF 09937; Figure 6. 

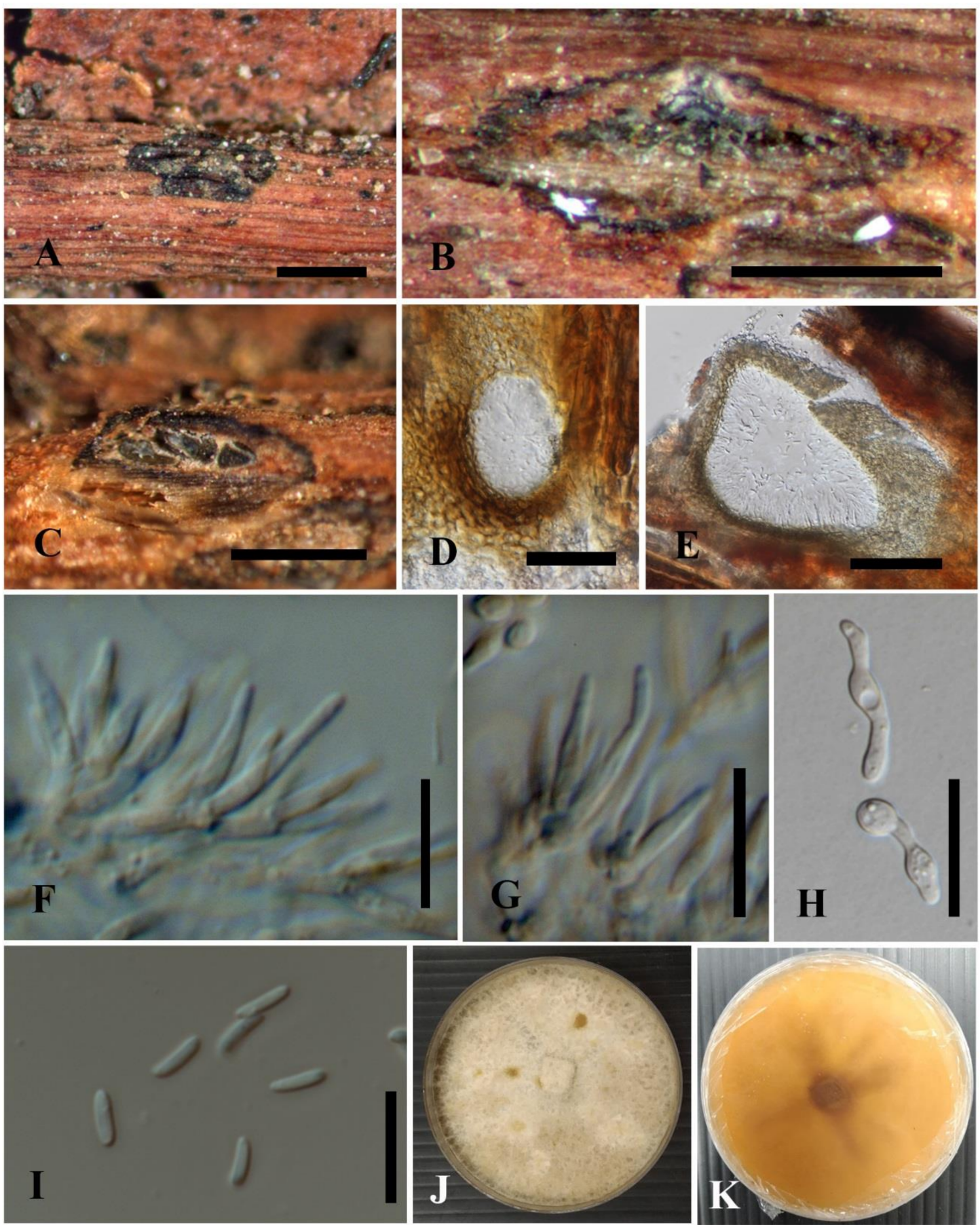

Figure 6. Cytospora phitsanulokensis (MFLU 21-0051, holotype). (A) Conidiomata on host substrate. (B,C) Longitudinal sections through conidioma. (D,E) Longitudinal sections of locules. (F,G) Conidiophores and conidiogenous cells with attached conidia. (H) Germinating conidia. (I) Conidia. (J,K) Colonies on PDA plate $(9 \mathrm{~cm}$ diam.) ((J) from above, (K) from reverse). Scale bars: $(\mathbf{A}-\mathbf{C})=500 \mu \mathrm{m},(\mathbf{D})=30 \mu \mathrm{m},(\mathbf{E})=50 \mu \mathrm{m},(\mathbf{F}-\mathbf{I})=10 \mu \mathrm{m}$.

Etymology: Name reflects the locality, Phitsanulok Province, Thailand, where the holotype was collected.

Holotype: MFLU 21-0051

Saprobic on unidentified decaying leaves (vein and petioles). Sexual morph: Undetermined. Asexual morph: Conidiomata $900-1100 \mu \mathrm{m}$ diam., pycnidial, semi-immersed in host tissue, solitary, erumpent, nearly flat, multi-loculate. Conceptacle black. Ectostromatic disc 
500-640 $\mu \mathrm{m}$ diam., brown to dark brown, circular to ovoid, one ostiole per disc. Ostioles inconspicuous, black. Peridium comprising few layers of cells of textura angularis, brown to dark brown. Locules numerous, irregular, subdivided frequently by invaginations with independent walls. Conidiophores hyaline, unbranched or occasionally branched at the bases, formed from the innermost layer of pycnidial wall, embedded in a gelatinous layer. Conidiogenous cells $6.7-14.1 \times 1.1-2.5 \mu \mathrm{m}(\bar{x}=9.8 \times 1.9 \mu \mathrm{m}, n=30)$, enteroblastic, phialidic, sub-cylindrical to cylindrical. Conidia 5.2-6.9 $\times 1.2-1.7 \mu \mathrm{m}(\bar{x}=6 \times 1.5 \mu \mathrm{m}, n=30)$, hyaline, unicellular, allantoid, guttulate, smooth, thin-walled.

Culture characteristics: Conidia germinating on PDA within $24 \mathrm{~h}$ germ tubes produced from both poles. Colonies on PDA reached at $8 \mathrm{~cm}$ diam. after 7 days at $28^{\circ} \mathrm{C}$, circular in shape, effuse, slightly raised, with entire margin, medium dense, floccose, white, in reverse pale yellowish to white.

Material examined: THAILAND, Phitsanulok, Wang Thong, on unidentified decaying leaves, 25 July 2019, E. Yasanthika, E5-2 (MFLU 21-0051, holotype), ex-type living culture, MFLUCC 21-0046.

Notes: Phylogenetically, Cytospora phitsanulokensis forms a distinct lineage and is closely related to C. acaciae, C. magnoliae and C. italica with 100\% ML, 100\% MP and 1.00 PP statistical support (Figure 1). Cytospora phitsanulokensis differs from C. italica by its larger conidiomata, shorter conidiogenous cell and longer conidia [51] (Table 4). Complete descriptions of $C$. acaciae and C. magnoliae were not available for morphological comparison [11,52]. Adam et al. [11] reported that Cytospora acaciae produces phialides with a long narrow channel of apical pores with lipid globules at one end of conidium; however, these characteristics were not observed in C. phitsanulokensis.

\subsection{Preliminary Screening of Antagonistic Activity against Fungal Pathogens}

Cytospora phitsanulokensis (MFLUCC 21-0046) exhibited the highest inhibition against Co. viniferum and F. sambucinum at $75.1 \pm 4.0 \%$ and $67.5 \pm 1.3 \%$, respectively (Table 5 , Figure 7). Cytospora shoreae (MFLUCC 21-0047 and MFLUCC 21-0048) showed a high inhibition effect against $C o$. viniferum at $64.0 \pm 1.5 \%$ and $61.8 \pm 1.4 \%$, respectively (Table 4 , Figure 7). However, Cytospora chiangmaiensis (MFLUCC 21-0049) had moderate inhibition activity against five tested plant pathogenic fungi (Table 5).

Table 5. In vitro antagonistic activity of Cytospora spp. against five plant pathogenic fungi after 10 days incubation at $28^{\circ} \mathrm{C}$.

\begin{tabular}{|c|c|c|c|c|}
\hline \multirow{2}{*}{ Fungal Name } & \multicolumn{4}{|c|}{ \% Growth Inhibition } \\
\hline & $\begin{array}{c}\text { Cytospora } \\
\text { chiangmaiensis } \\
\text { (MFLUCC 21-0049) }\end{array}$ & $\begin{array}{c}\text { C. shoreae } \\
\text { (MFLUCC 21-0047) }\end{array}$ & $\begin{array}{c}\text { C. shoreaeb } \\
\text { (MFLUCC 21-0048) }\end{array}$ & $\begin{array}{l}\text { C. phitsanulokensis } \\
\text { (MFLUCC 21-0046) }\end{array}$ \\
\hline $\begin{array}{l}\text { Colletotrichum fructicola } \\
\text { (MFLUCC 18-1160) }\end{array}$ & $44.2 \pm 2.5^{a}$ & $49.6 \pm 0.0^{\mathrm{a}}$ & $50.5 \pm 5.7^{a}$ & $37.4 \pm 1.5^{\mathrm{a}}$ \\
\hline $\begin{array}{c}\text { Co. siamense } \\
\text { (MFLUCC 18-1162) }\end{array}$ & $18.1 \pm 4.4^{\mathrm{a}}$ & $31.9 \pm 0.4^{b}$ & $53.5 \pm 1.8^{c}$ & $39.5 \pm 2.2^{b}$ \\
\hline $\begin{array}{l}\text { Co. artocarpicola } \\
\text { (MFLUCC 18-1167) }\end{array}$ & $11.7 \pm 3.9^{\mathrm{a}}$ & $14.9 \pm 5.5^{\mathrm{a}}$ & $32.3 \pm 3.6^{b}$ & $58.0 \pm 1.0^{\mathrm{c}}$ \\
\hline $\begin{array}{c}\text { Co. viniferum } \\
\text { (MFLUCC 18-1179) }\end{array}$ & $30.0 \pm 3.9^{a}$ & $64.0 \pm 1.5^{b}$ & $61.8 \pm 1.4^{b}$ & $75.1 \pm 4.0^{b}$ \\
\hline $\begin{array}{l}\text { Fusarium sambucinum } \\
\text { (MFLUCC 17-1056) }\end{array}$ & $17.4 \pm 3.2^{\mathrm{a}}$ & $39.1 \pm 4.2^{b}$ & $33.1 \pm 4.4^{b}$ & $67.5 \pm 1.3^{c}$ \\
\hline
\end{tabular}

The values represent I $\% \pm$ S.E. Different letters indicate significant differences between tested pathogens $(p \leq 0.05)$ according to Tukey's HSD test. The values greater than $60 \%$ with clear inhibition zone are bold. 

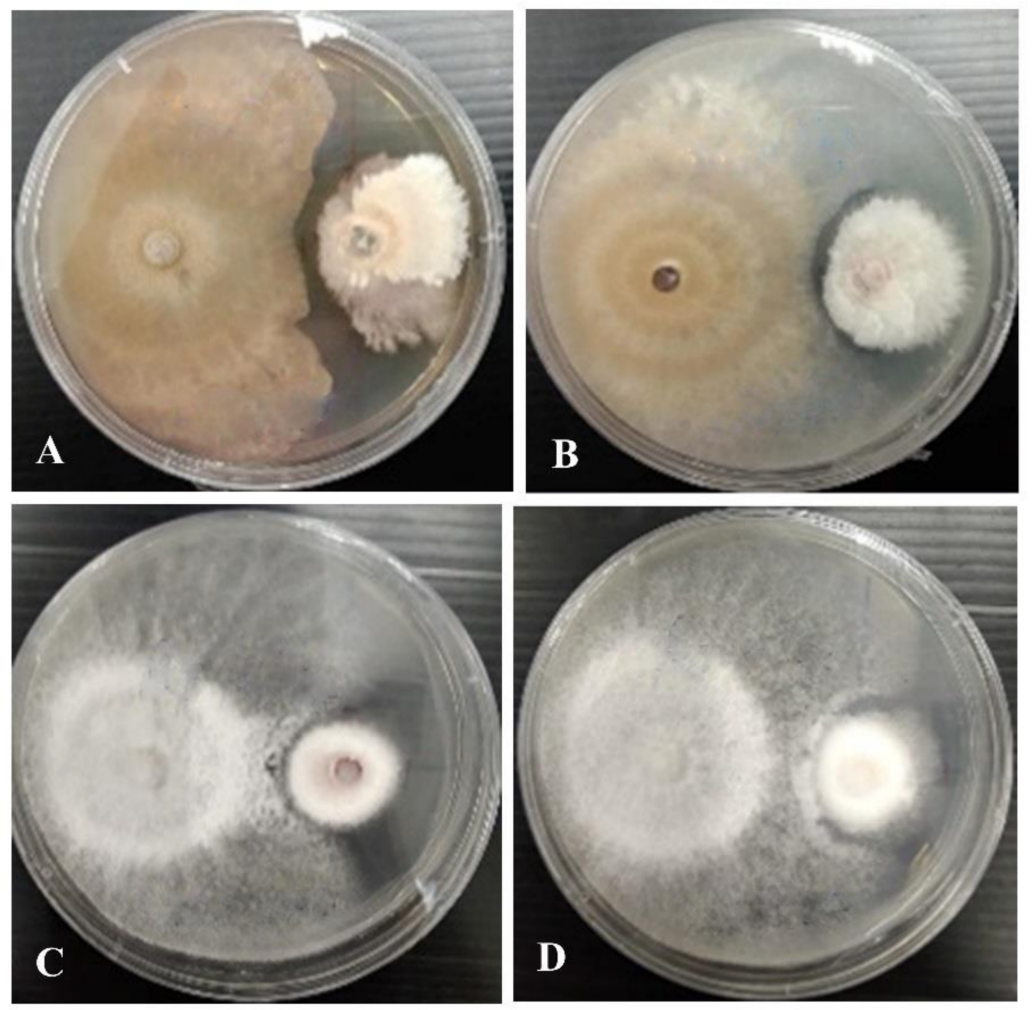

Figure 7. The antagonistic activity of Cytospora species (left colonies) against fungal pathogens (right colonies). (A) Cytospora shoreae (MFLUCC 21-0047) vs. Colletotrichum viniferum (MFLUCC 18-1179). (B) C. shoreae (MFLUCC 21-0048) vs. Co. viniferum (MFLUCC 18-1179). (C) C. phitsanulokensis (MFLUCC 21-0046) vs. Co. viniferum (MFLUCC 18-1179). (D) C. phitsanulokensis (MFLUCC 21-0046) vs. Fusarium sambucinum (MFLUCC 17-1056).

\section{Discussion}

This study provides taxonomic novelties of Cytospora species discovered from Thailand and their antagonistic activities against fungal pathogens. We show that six-locus phylogeny (ITS, LSU, ACT, RPB2, TEF1- $\alpha$ and TUB2) facilitates species delineation in Cytospora which is consistent with previous studies $[1,3,17,18]$. In addition, Cytospora chiangmaiensis and $C$. shoreae are phylogenetically closely related to $C$. diopuiensis, which was also collected from Chiang Mai, Thailand [2], indicating the close geographical relationship of these taxa. Based on the Fungus-Host USDA database [53], two new species, Cytospora chiangmaiensis and C. shoreae, are recorded for the first time on Shorea sp. (Dipterocarpaceae). Cytospora phitsanulokensis collected from Phitsanulok, Thailand, is phylogenetically closely related to C. acaciae, C. italica and C. magnoliae. The GCPSR analyses provided evidence for Cytospora phitsanulokensis as a separate species. However, the presence of recombination among strains of C. acaciae, C. italica and C. magnoliae shown in the spilt graph (Figure 2) may be caused by the lack of some gene regions in the dataset. Thus, more strains and new sequence data of $C$. italica and $C$. magnoliae should be obtained to better confirm their taxonomic placements in the $C$. acaciae clade.

Cytospora species are discovered in woody substrates such as bark, branches and twigs [1]. It should be noted that the stromata of Cytospora were only detected on vein and petioles, but not on leaf lamina for all our samples. Adams et al. [11] mentioned that the formation of stroma of Cytospora could possibly decrease in leaves compared to bark. Cytospora species are known as saprobes on dead plants and are one of the important plant pathogens causing dieback and canker diseases on a wide range of hosts [1-3,5,9,16-18,23]. Considering that the species number of Cytospora is ever-increasing and related taxonomic knowledge continues to expand [1], more extensive sampling of both fresh and dead plant samples in selected hosts and locations should be implemented to improve and stabilize 
the identification and classification of Cytospora species. In addition, Cytospora are interesting fungal taxa for screening potential biological activities $[24,25,27]$. Previous studies reported novel bioactive compounds produced by Cytospora spp. [24,25,27,54]; however, species identification of these potential strains was not established. The taxonomic classification and phylogenetic relationships can be used as important tools for the screening of biologically active strains and their biological activities for further applications [55,56].

In this study, we identified new Cytospora species and demonstrated that different strains and/or species can inhibit fungal pathogens. However, our study only conducted a preliminary screening of Cytospora that can inhibit fungal pathogens, using the dual culture method. Although our new species were identified as saprobes based on their occurrence on decaying leaf substrates, their pathogenicity should be clarified, as this genus is well known for pathogens. Therefore, to confirm whether our Cytospora strains are true saprobes, further research is needed. Further studies will be carried out in the near future to check the pathogenicity of the new Cytospora species, elucidate the biology of these fungi and screen secondary metabolites.

Author Contributions: Conceptualization, J.M., A.M. (Ausana Mapook) and C.N.; Methodology, J.M., S.T., A.M. (Areerat Manowong), A.M. (Ausana Mapook) and C.N.; Formal analysis, J.M. and C.N.; Data curation, J.M.; Resources, J.M., S.T., K.D.H. and I.P.; Supervision, K.D.H. and I.P.; Writingoriginal draft, J.M., S.T., A.M. (Areerat Manowong), A.M. (Ausana Mapook), C.N., K.D.H. and I.P.; Writing —review and editing, J.M., S.T., A.M. (Ausana Mapook), C.N., K.D.H. and I.P. All authors have read and agreed to the published version of the manuscript.

Funding: Kevin D. Hyde thanks the Thailand Research Fund "Impact of climate change on fungal diversity and biogeography in the Greater Mekong Subregion" (project no. RDG6130001) for supporting this research. Saowaluck Tibpromma would like to thank the Inter-national Postdoctoral Exchange Fellowship Program (number Y9180822S1), CAS President's International Fellowship Initiative (PIFI) (number 2020PC0009), China Postdoctoral Science Foundation and Yunnan Human Resources, and the Social Security Department Foundation for fund-ing her postdoctoral research.

Informed Consent Statement: Not applicable.

Data Availability Statement: Data can be found within the manuscript.

Acknowledgments: Jutamart Monkai would like to thank the Postdoctoral Fellowship from Mae Fah Luang University. Jutamart Monkai is grateful to Rungtiwa Phookamsak, Ruvishika Jayawardena, Naruemon Huanraluek, Erandi Yasanthika, Guang-Cong Ren, Gao Ying and Mark S. Calabon for their assistance during this research. Shaun Pennycook from Landcare Research, Auckland, New Zealand, is thanked for advising on the taxon name. Austin Smith at World Agroforestry (ICRAF), Kunming Institute of Botany, China, is thanked for English editing. We also acknowledge the Biology Experimental Center, Germplasm Bank of Wild Species, Kunming Institute of Botany, Chinese Academy of Sciences for providing the molecular laboratory facilities. Itthayakorn Promputtha is grateful to Chiang Mai University for partial support this research.

Conflicts of Interest: The authors declare no conflict of interest.

\section{References}

1. Fan, X.; Bezerra, J.D.P.; Tian, C.; Crous, P. Cytospora (Diaporthales) in China. Persoonia-Mol. Phylogeny Evol. Fungi 2020, 45, 1-45. [CrossRef] [PubMed]

2. Shang, Q.J.; Hyde, K.D.; Camporesi, E.; Maharachchikumbura, S.S.N.; Norphanphoun, C.; Brooks, S.; Liu, J.K. Additions to the genus Cytospora with sexual morph in Cytosporaceae. Mycosphere 2020, 11, 189-224. [CrossRef]

3. Zhu, H.; Pan, M.; Bezerra, J.D.P.; Tian, C.; Fan, X. Discovery of Cytospora species associated with canker disease of tree hosts from Mount Dongling of China. MycoKeys 2020, 62, 97-121. [CrossRef]

4. Maharachchikumbura, S.S.N.; Hyde, K.D.; Jones, E.B.G.; McKenzie, E.H.C.; Bhat, J.D.; Dayarathne, M.C.; Huang, S.-K.; Norphanphoun, C.; Senanayake, I.C.; Perera, R.H.; et al. Families of Sordariomycetes. Fungal Divers. 2016, 79, 1-317. [CrossRef]

5. Norphanphoun, C.; Raspé, O.; Jeewon, R.; Wen, T.-C.; Hyde, K.D. Morphological and phylogenetic characterisation of novel Cytospora species associated with mangroves. MycoKeys 2018, 38, 93-120. [CrossRef]

6. $\quad$ Ehrenberg, C.G. Sylvae Mycologicae Berolinenses; Formis Teophili Bruschcke: Berlin, Germany, 1818; pp. 1-32.

7. Donk, M.A. Nomina conservanda proposita I. Propos. Fungi. Deuteromycetes Regnum Veg. 1964, 34, 7-15. 
8. Rossman, A.Y.; Crous, P.W.; Hyde, K.D.; Hawksworth, D.L.; Aptroot, A.; Bezerra, J.L.; Bhat, J.D.; Boehm, E.; Braun, U.; Boonmee, S.; et al. Recommended names for pleomorphic genera in Dothideomycetes. IMA Fungus 2015, 6, 507-523. [CrossRef]

9. Norphanphoun, C.; Doilom, M.; Daranagama, D.A.; Phookamsak, R.; Wen, T.C.; Bulgakov, T.S.; Hyde, K.D. Revisiting the genus Cytospora and allied species. Mycosphere 2017, 8, 51-97. [CrossRef]

10. McNeill, J.; Barrie, F.R.; Buck, W.R.; Demoulin, V.; Greuter, W.; Hawksworths, D.L.; Herendeen, P.S.; Knapp, S.; Marhold, K.; Prado, J.; et al. International code of nomenclature for algae, fungi and plants (Melbourne Code) adopted by the Eighteenth International Botanical Congress Melbourne, Australia, July 2011. Regnum Veg. 2012, 154, 1-140.

11. Adams, G.C.; Wingfield, M.J.; Common, R.; Roux, J. Phylogenetic relationships and morphology of Cytospora species and related teleomorphs (Ascomycota, Diaporthales, Valsaceae) from Eucalyptus. Stud. Mycol. 2005, 52, 1-144.

12. Wang, X.; Wei, J.; Huang, L.; Kang, Z. Re-evaluation of pathogens causing Valsa canker on apple in China. Mycologia 2011, 103, 317-324. [CrossRef]

13. Fan, X.; Hyde, K.D.; Liu, M.; Liang, Y.; Tian, C. Cytospora species associated with walnut canker disease in China, with description of a new species C. gigalocus. Fungal Biol. 2015, 119, 310-319. [CrossRef] [PubMed]

14. Hyde, K.D.; Hongsanan, S.; Jeewon, R.; Bhat, D.J.; McKenzie, E.H.C.; Jones, E.B.G.; Phookamsak, R.; Ariyawansa, H.; Boonmee, S.; Zhao, Q.; et al. Fungal diversity notes 367-490: Taxonomic and phylogenetic contributions to fungal taxa. Fungal Divers. 2016, 80, 1-270. [CrossRef]

15. Lawrence, D.P.; Holland, L.A.; Nouri, M.T.; Travadon, R.; Abramians, A.; Michailides, T.J.; Trouillas, F.P. Molecular phylogeny of Cytospora species associated with canker diseases of fruit and nut crops in California, with the descriptions of ten new species and one new combination. IMA Fungus 2018, 9, 333-369. [CrossRef] [PubMed]

16. Jiang, N.; Yang, Q.; Fan, X.-L.; Tian, C.-M. Identification of six Cytospora species on Chinese chestnut in China. MycoKeys 2020, 62, 1-25. [CrossRef]

17. Pan, M.; Zhu, H.; Bonthond, G.; Tian, C.; Fan, X. High diversity of Cytospora associated with canker and dieback of Rosaceae in China, with 10 new species described. Front. Plant. Sci. 2020, 11, 690. [CrossRef]

18. Pan, M.; Zhu, H.; Tian, C.; Huang, M.; Fan, X. Assessment of Cytospora isolates from conifer cankers in china, with the descriptions of four new Cytospora species. Front. Plant. Sci. 2021, 12. [CrossRef]

19. Index Fungorum. Available online: http:/ /www.indexfungorum.org (accessed on 1 August 2021).

20. Hyde, K.D.; Norphanphoun, C.; Maharachchikumbura, S.S.N.; Bhat, D.J.; Jones, E.B.G.; Bundhun, D.; Chen, Y.J.; Bao, D.F.; Boonmee, S.; Calabon, M.S.; et al. Refined families of Sordariomycetes. Mycosphere 2020, 11, 305-1059. [CrossRef]

21. Senanayake, I.; Crous, P.; Groenewald, J.; Maharachchikumbura, S.; Jeewon, R.; Phillips, A.; Bhat, J.; Perera, R.; Li, Q.; Li, W.; et al Families of Diaporthales based on morphological and phylogenetic evidence. Stud. Mycol. 2017, 86, 217-296. [CrossRef]

22. Adams, G.C.; Roux, J.; Wingfield, M.J. Cytospora species (Ascomycota, Diaporthales, Valsaceae): Introduced and native pathogens of trees in South Africa. Australas. Plant. Pathol. 2006, 35, 521-548. [CrossRef]

23. Wang, Y.-L.; Lu, Q.; Decock, C.; Li, Y.-X.; Zhang, X.-Y. Cytospora species from Populus and Salix in China with C. davidiana sp. nov. Fungal Biol. 2015, 119, 420-432. [CrossRef] [PubMed]

24. Brady, S.F.; Wagenaar, M.M.; Singh, M.P.; Janso, J.E.; Clardy, J. The Cytosporones, new octaketide antibiotics isolated from an endophytic fungus. Org. Lett. 2000, 2, 4043-4046. [CrossRef] [PubMed]

25. Singh, M.P.; Janso, J.E.; Brady, S.F. Cytoskyrins and cytosporones produced by Cytospora sp. CR200: Taxonomy, fermentation and biological activities. Mar. Drugs 2007, 5, 71-84. [CrossRef] [PubMed]

26. Sadorn, K.; Saepua, S.; Boonyuen, N.; Boonruangprapa, T.; Rachtawee, P.; Pittayakhajonwut, P. Antimicrobial activity and cytotoxicity of xanthoquinodin analogs from the fungus Cytospora eugeniae BCC42696. Phytochemistry 2018, 151, 99-109. [CrossRef]

27. Deng, Q.; Li, G.; Sun, M.; Yang, X.; Xu, J. A new antimicrobial sesquiterpene isolated from endophytic fungus Cytospora sp. from the Chinese mangrove plant Ceriops tagal. Nat. Prod. Res. 2018, 34, 1404-1408. [CrossRef]

28. Senanayake, I.C.; Rathnayaka, A.R.; Marasinghe, D.S.; Calabon, M.S.; Gentekaki, E.; Lee, H.B.; Hurdeal, V.G.; Pem, D.; Dissanayake, L.S.; Wijesinghe, S.N.; et al. Morphological approaches in studying fungi: Collection, examination, isolation, sporulation and preservation. Mycosphere 2020, 11, 2678-2754. [CrossRef]

29. Jayasiri, S.C.; Hyde, K.D.; Ariyawansa, H.; Bhat, J.; Buyck, B.; Cai, L.; Dai, Y.-C.; Abd-Elsalam, K.A.; Ertz, D.; Hidayat, I.; et al. The faces of fungi database: Fungal names linked with morphology, phylogeny and human impacts. Fungal Divers. 2015, 74, 3-18. [CrossRef]

30. White, T.J.; Bruns, S.; Lee, S.; Taylor, J. Amplification and direct sequencing of fungal ribosomal RNA genes for phylogenetics. PCR Protoc. A Guid. to Methods Appl. 1990, 18, 315-322.

31. Vilgalys, R.; Hester, M. Rapid genetic identification and mapping of enzymatically amplified ribosomal DNA from several Cryptococcus species. J. Bacteriol. 1990, 172, 4238-4246. [CrossRef]

32. Liu, Y.J.; Whelen, S.; Hall, B.D. Phylogenetic relationships among ascomycetes: Evidence from an RNA polymerse II subunit. Mol. Biol. Evol. 1999, 16, 1799-1808. [CrossRef]

33. Carbone, I.; Kohn, L.M. A method for designing primer sets for speciation studies in filamentous ascomycetes. Mycologia 1999, 91, 553-556. [CrossRef]

34. Glass, N.L.; Donaldson, G.C. Development of primer sets designed for use with the PCR to amplify conserved genes from filamentous ascomycetes. Appl. Environ. Microbiol. 1995, 61, 1323-1330. [CrossRef] [PubMed] 
35. Katoh, K.; Rozewicki, J.; Yamada, K.D. MAFFT online service: Multiple sequence alignment, interactive sequence choice and visualization. Briefings Bioinform. 2017, 20, 1160-1166. [CrossRef]

36. Capella-Gutierrez, S.; Silla-Martinez, J.M.; Gabaldon, T. TrimAl: A tool for automated alignment trimming in large-scale phylogenetic analyses. Bioinformatics 2009, 25, 1972-1973. [CrossRef] [PubMed]

37. Miller, M.A.; Pfeiffer, W.; Schwartz, T. Creating the CIPRES science gateway for inference of large phylogenetic trees. In Proceedings of the 2010 Gateway Computing Environments Workshop (GCE), New Orleans, LA, USA, 14 November 2010; IEEE: New Orleans, LA, USA, 2010; pp. 1-8.

38. Swofford, D.L. PAUP* Phylogenetic Analysis Using Parsimony * (and Other methods); Version 4.0; Sinauer Associates: Sunderland, UK, 2002.

39. Nylander, J.A. MrModeltest 2. Program Distributed by the Author. Department of Systematic Zoology; Evolutionary Biology Centre, Uppsala University: Uppsala, Sweden, 2004.

40. Huelsenbeck, J.P.; Ronquist, F. MRBAYES: Bayesian inference of phylogenetic trees. Bioinformatics 2001, 17, 754-755. [CrossRef]

41. Zhaxybayeva, O.; Gogarten, J.P. Bootstrap, Bayesian probability and maximum likelihood mapping: Exploring new tools for comparative genome analyses. BMC Genomic. 2002, 3, 1-15. [CrossRef]

42. Ronquist, F.; Teslenko, M.; Van Der Mark, P.; Ayres, D.L.; Darling, A.; Hoehna, S.; Larget, B.; Liu, L.; Suchard, M.A.; Huelsenbeck, J.P. MrBayes 3.2: Efficient bayesian phylogenetic inference and model choice across a large model space. Syst. Biol. 2012, 61, 539-542. [CrossRef] [PubMed]

43. Rambaut, A.; Drummond, A. Tracer v1.4. Available online: http:/ / beast.bio.ed.ac.uk/Tracer (accessed on 3 August 2021).

44. Rambaut, A.; Drummond, A.J. FigTree: Tree Figure Drawing Tool; Institute of Evolutionary Biology, University of Edinburgh: Edinburgh, Scotland, 2012.

45. Bruen, T.C.; Philippe, H.; Bryant, D. A simple and robust statistical test for detecting the presence of recombination. Genetics 2006, 172, 2665-2681. [CrossRef]

46. Quaedvlieg, W.; Binder, M.; Groenewald, J.; Summerell, B.; Carnegie, A.; Burgess, T.; Crous, P. Introducing the consolidated species concept to resolve species in the Teratosphaeriaceae. Persoonia-Mol. Phylogeny Evol. Fungi 2014, 33, 1-40. [CrossRef]

47. Huson, D.H. SplitsTree: Analyzing and visualizing evolutionary data. Bioinformatics 1998, 14, 68-73. [CrossRef]

48. Huson, D.H.; Bryant, D. Application of phylogenetic networks in evolutionary studies. Mol. Biol. Evol. 2005, 23, 254-267. [CrossRef]

49. Hamzah, T.N.T.; Lee, S.Y.; Hidayat, A.; Terhem, R.; Faridah-Hanum, I.; Mohamed, R. Diversity and characterization of endophytic fungi isolated from the tropical mangrove species, Rhizophora mucronata, and identification of potential antagonists against the soil-borne fungus, Fusarium solani. Front. Microbiol. 2018, 9, 1707. [CrossRef] [PubMed]

50. Jeewon, R.; Hyde, K.D. Establishing species boundaries and new taxa among fungi: Recommendations to resolve taxonomic ambiguities. Mycosphere 2016, 7, 1669-1677. [CrossRef]

51. Thambugala, K.M.; Daranagama, D.A.; Phillips, A.; Bulgakov, T.; Bhat, D.J.; Camporesi, E.; Bahkali, A.H.; Eungwanichayapant, P.D.; Liu, Z.-Y.; Hyde, K.D. Microfungi on Tamarix. Fungal Divers 2016, 82, 239-306. [CrossRef]

52. Kepley, J.B.; Reeves, F.B.; Jacobi, W.R.; Adams, G.C. Species associated with Cytospora canker on Populus tremuloides. Mycotaxon 2015, 130, 783-805. [CrossRef]

53. Farr, D.F.; Rossman, A.Y. Fungal Databases, U.S. National Fungus Collections, ARS, USDA. Available online: https://nt.ars-grin gov/fungaldatabases / (accessed on 10 August 2021).

54. Wei, C.; Deng, Q.; Sun, M.; Xu, J. Cytospyrone and cytospomarin: Two new polyketides isolated from mangrove endophytic fungus, Cytospora sp. Molecules 2020, 25, 4224. [CrossRef] [PubMed]

55. Raja, H.A.; Miller, A.N.; Pearce, C.J.; Oberlies, N.H. Fungal identification using molecular tools: A primer for the natural products research community. J. Nat. Prod. 2017, 80, 756-770. [CrossRef]

56. Doilom, M.; Guo, J.-W.; Phookamsak, R.; Mortimer, P.E.; Karunarathna, S.C.; Dong, W.; Liao, C.-F.; Yan, K.; Pem, D.; Suwannarach, N.; et al. Screening of phosphate-solubilizing fungi from air and soil in Yunnan, China: Four novel species in Aspergillus, Gongronella, Penicillium, and Talaromyces. Front. Microbiol. 2020, 11, 585215. [CrossRef] 